\title{
PhSeBr-Catalyzed Selective Addition of Thiols to $\alpha, \beta$-Unsaturated Carbonyl Compounds: Regioselective Synthesis of Thioacetals vs. $\beta$-Mercapto Ketones
}

\author{
Caroline C. Schneider, ${ }^{a}$ Flávia Manarin, ${ }^{a}$ Rodrigo B. Panatieri, ${ }^{b}$ \\ Olga S. R. Barros ${ }^{c}$ and Gilson Zeni ${ }^{*, a}$
}

${ }^{a}$ Laboratório de Síntese, Reatividade, Avaliação Farmacológica e Toxicidade de Organocalcogênios, CCNE, UFSM, 97105-900 Santa Maria-RS, Brazil

${ }^{b}$ Universidade Federal de Uberlândia, Faculdade de Ciências Integradas do Pontal-Ituiutaba, 38302-000 Ituiutaba-MG, Brazil

'Instituto de Química, Universidade Federal de Góias, 74001-970 Goiania-GO, Brazil

\begin{abstract}
Apresentamos aqui nossos resultados da adição de tióis, catalisada por $\mathrm{PhSeBr}$, a compostos carbonílicos $\alpha, \beta$-insaturados sob condições brandas para obter regiosseletivamente $\beta$-mercapto cetonas ou tioacetais com altos rendimentos e seletividade. A reação foi principalmente controlada pela temperatura, na qual os produtos de adição 1,4 foram obtidos à temperatura de $-20{ }^{\circ} \mathrm{C}$. Inversamente quando a reação foi realizada sob refluxo, tioacetais foram obtidos como único produto. $\mathrm{O}$ método admite diversos grupos funcionais, como alquilicos, benzilicos e arilicos com substituintes neutros, deficientes e ricos em elétrons no anel aromático.
\end{abstract}

We present herein results on the $\mathrm{PhSeBr}$-catalyzed addition of thiols to $\alpha, \beta$-unsaturated carbonyl compounds under mild conditions to afford regioselectivily $\beta$-mercapto ketones or thioacetals in high yields and selectivity. The reaction was highly controlled by the temperature in which, the 1,4-addition products were obtained when the temperature was $-20^{\circ} \mathrm{C}$, conversely when the reaction was carried out at reflux, the thioacetals were obtained as a sole product. The developed protocol stands a wide range of functional groups, in which alkyl, benzyl and aryl with neutral, electron deficient and electron rich substituents on the aromatic ring.

Keywords: thioacetals, $\beta$-mercapto ketones, chalcogenides

\section{Introduction}

Thioacetals are useful intermediates in organic synthesis and are often used as masked carbonyl groups, in particular $\alpha$-lithiated thioacetals that are synthetic equivalents of carbonyl anions. ${ }^{1,2}$ Thioacetals and thioketals are particularly attractive as carbonyl protecting groups in complex molecule synthesis because of their added stability to acidic conditions. In this view, there have been continued improvements in the thioacetal synthesis methods. Usually, these compounds are prepared by protic or Lewis acid-catalyzed condensation of carbonyl compounds with thiols. Lewis acid catalysts such as $\mathrm{ZnCl}_{2}$, $\mathrm{LnCl}_{3},{ }^{4} \mathrm{FeCl}_{3} / \mathrm{SiO}_{2},{ }^{5} \mathrm{AlCl}_{3},{ }^{6} \mathrm{ZrCl}_{4} / \mathrm{SiO}_{2}{ }^{7} \mathrm{TeCl}_{4},{ }^{8} \mathrm{SnCl}_{2},{ }^{9}$ $\mathrm{SiCl}_{4},{ }^{10} \mathrm{TiCl}_{4},{ }^{11} \mathrm{BF}_{3} \cdot \mathrm{OEt}_{2}{ }^{12}$ and others methods ${ }^{13-15}$ have been used for this purpose. Despite those methods reported

\footnotetext{
*e-mail: gzeni@pq.cnpq.br
}

in the literature, some problems were found as, difficulties in work-up, isolation, requirement of inert atmosphere, harsh reaction conditions, expensive and stoichiometric reagents, incompatibility with other protecting groups and failure to protect desactivated and hindered substrates. In contrast, none of the reported methods describe the selective catalytic preparation of $\beta$-mercapto ketones or thioacetals from $\alpha, \beta$-unsaturated carbonyl compounds with thiols, catalyzed by $\mathrm{PhSeBr}$. Therefore, it would be of interest to define a method in order to prepare selectively $\beta$-mercapto ketones or thioacetals starting from $\alpha, \beta$-unsaturated carbonyl compounds and thiols (Scheme 1).

\section{Results and Discussion}

Our initial studies have focused on the development of an optimum set of reaction conditions to obtain $\beta$-mercapto ketones in the absence of thioacetals. In 


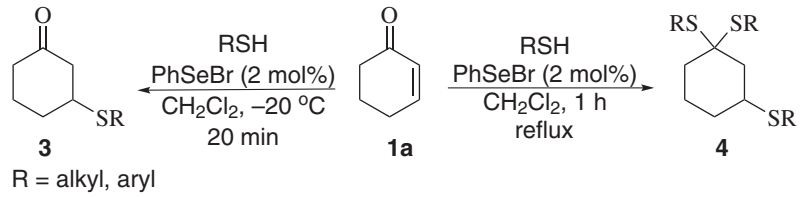

Scheme 1. General scheme.

this way, cyclohex-2-enone 1a and benzenethiol 2a were used as standard substrates and the variation in the temperature, time, presence or absence of catalyst were investigated. At first, the reaction was tested under room temperature, using $\mathrm{CH}_{2} \mathrm{Cl}_{2}$ as solvent in the presence of $\mathrm{PhSeBr}(2 \mathrm{~mol} \%)$; thus after 10 min the product of 1,4 -addition was obtained in $65 \%$ yield. However, under this reaction condition traces of the thioacetal were also obtained. In attempt to avoid the thioacetal by-product and to select the $\beta$-mercapto ketones, as a sole product, we carried out the reaction at 0 and $-20{ }^{\circ} \mathrm{C}$. At $0{ }^{\circ} \mathrm{C}$ a mixture of both $\beta$-mercapto ketone and thioacetal was yet obtained, but changing the temperature to $-20{ }^{\circ} \mathrm{C}$, after $20 \mathrm{~min}$, the desired product in $79 \%$ yield was found with no mixture. Regarding the influence of the catalyst, neither $\beta$-mercapto ketones nor thioacetals

Table 1. Addition of thiols in the carbonyl group; synthesis of $\beta$-mercapto ketones

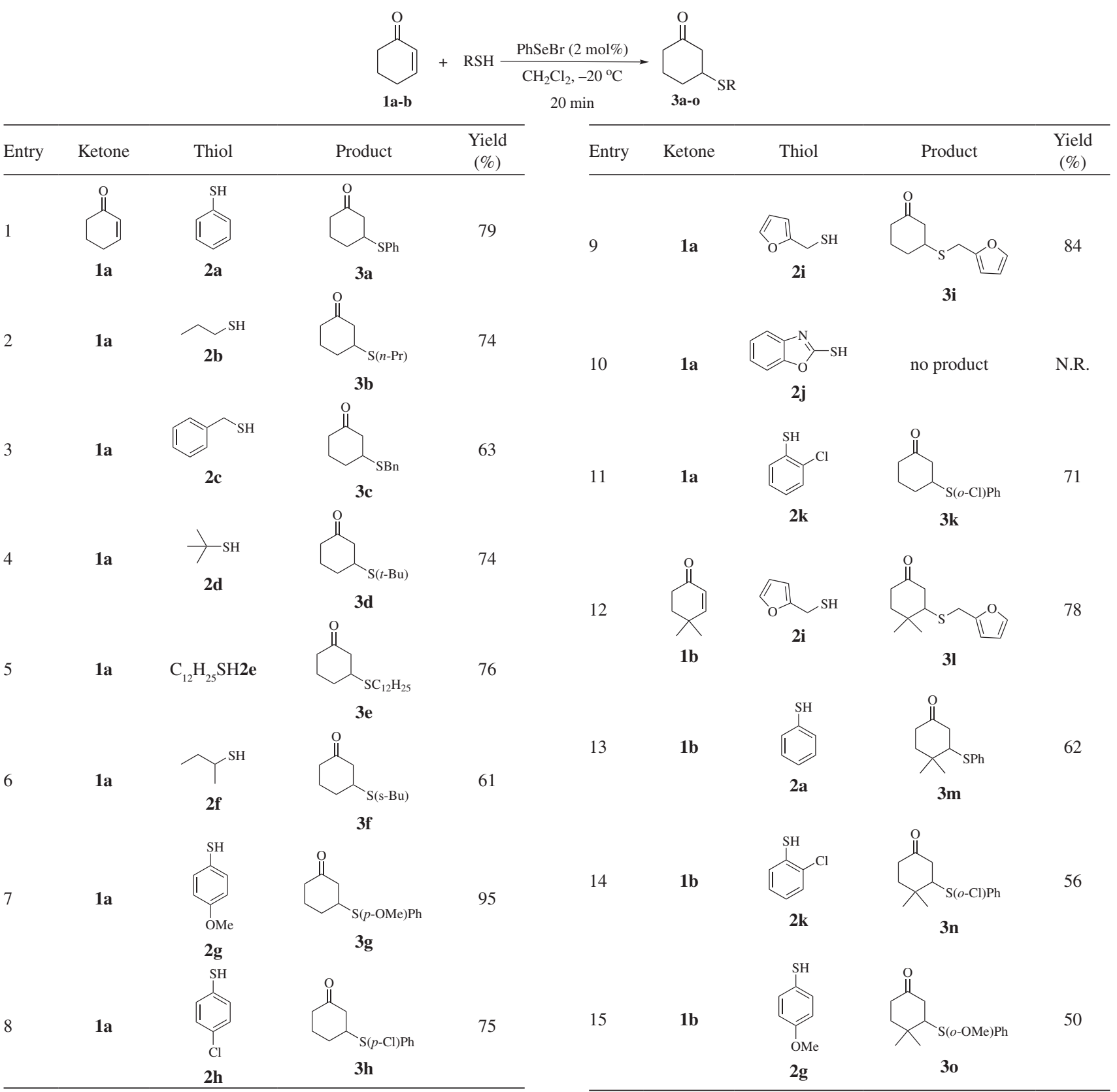

N.R.: no reaction. 
were obtained when the reactions was carried out in the absence of PhSeBr. Thus, the analysis of the optimized reaction conditions demonstrated that the optimal ones for this procedure were the addition of cyclohex-2-enone 1a $(1 \mathrm{mmol})$, benzenethiol 2a $(1.1 \mathrm{mmol}), \mathrm{PhSeBr}$ (2 $\mathrm{mol} \%)$ and $\mathrm{CH}_{2} \mathrm{Cl}_{2}(1 \mathrm{~mL})$ as a solvent. After the addition, the reaction was stirred for $20 \mathrm{~min}$ at $-20^{\circ} \mathrm{C}$ and the product $3 \mathbf{a}$ was obtained in $79 \%$ yield. These reactions conditions were systematically applied to other substrates to demonstrate the efficiency of this method, and the results are summarized in Table 1.

Inspections of Table 1 show that in general, all of the reactions proceeded smoothly with good yields. Most importantly, the addition turned out to be general with respect to a diverse array of functional thiol sources. Our experiments showed that the reaction with thiols having aryl and aryl substituted, was sensitive to the electronic nature of functional groups present in the aromatic ring. Electron donating group such as metoxy gave the conjugated addition product in high yield $95 \%$, in contrast to this, electron withdrawing group decrease the yield (Table 1, entries 7, 8 and 11). We also observed that hindered and non-hindered alkyl thiols gave the desired products in good yields (Table 1; entries 2, 4 and 6). As shown in Table 1, bulky carbonyl ketone afforded the 1,4-addition product in moderated yields (Table 1, entries 13-15). A limitation

Table 2. Addition of thiols in the carbonyl group; synthesis of thioacetals Aqui corrigir só o $1 \mathrm{~h}$

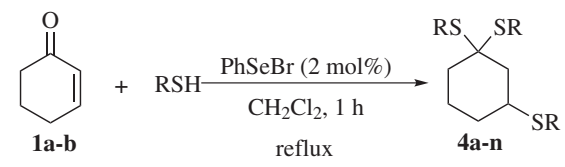

Entry

\begin{tabular}{lcccc}
\hline Entry & ketone & Thiol & Product & $\begin{array}{c}\text { Yield } \\
(\%)\end{array}$ \\
\hline 8 & $\mathbf{1 a}$ &
\end{tabular}

9

10

1a

$1 a$

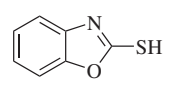

no product

4i

$2 \mathrm{j}$

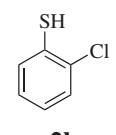

2k

11

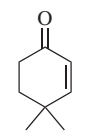

${ }_{\mathbf{2 i}}$

1b

12

$1 b$
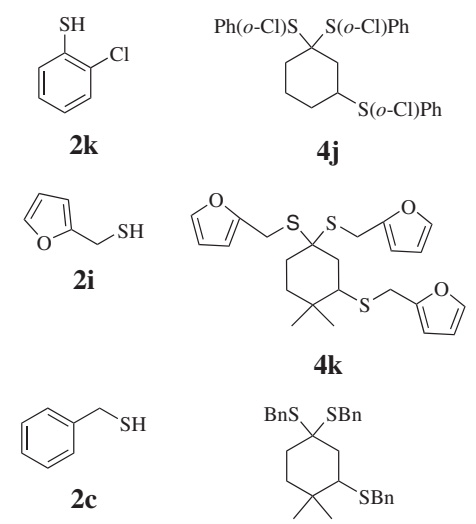

$13 \quad \mathbf{1 b}$

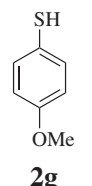

14

$1 b$

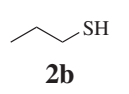

Ph(Mep-O)S $\quad$ S(p-OMe)Ph

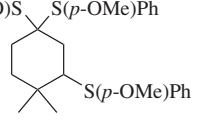

$4 \mathrm{~m}$

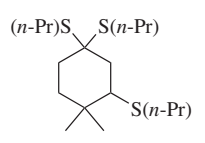

$4 k$

41

78

N.R. 
in this methodology was observed when oxazolethiol derivative $\mathbf{2} \mathbf{j}$ was used as thiol source. In this case no product was obtained, even under long reaction time, probably due to steric effects.

It was gratifying to discover the use of cyclohex-2enone 1a and benzenethiol $\mathbf{2} \mathbf{a}$ as standard substrates and the simply changing in the temperature from $-20{ }^{\circ} \mathrm{C}$ to reflux, in the procedure described to obtaine $\beta$-mercapto ketones $\mathbf{3}$, had a dramatic effect. Thus, the reaction of cyclohex-2-enone $1 \mathbf{a}(1 \mathrm{mmol})$, benzenethiol $2 \mathbf{a}$ (4.0 equiv.) with $\mathrm{PhSeBr}(2 \mathrm{~mol} \%)$ in $\mathrm{CH}_{2} \mathrm{Cl}_{2}(1 \mathrm{~mL})$ at reflux, gave thioacetal $\mathbf{4 a}$ as the sole product in $85 \%$ yield. Using the optimized reaction conditions, a wide variety of thiols containing useful functional groups can be successfully used as substrate (Table 2). The results revealed that the aryl, alkyl and benzyl thiols, gave the product efficiently under these conditions. The exception was the bulky thiols $\mathbf{2 d}, \mathbf{2 J}$ and $\mathbf{2 k}$, which did not gave the desired product, even changing the reaction conditions.

The fact that the protection of thiols has found widespread applications in the organic transformations ${ }^{16}$ associated with the simple preparation, low cost and easy handling of $\mathrm{PhSeBr}$, prompted us to study its application on the thiol reaction with dihydropyrane. Thus, the standard reaction condition applied to prepare the $\beta$-mercapto ketones $\mathbf{3}$ was also tested for the reaction of thiols and dihydropyrane. In this way, the reaction of

Table 3 Addition of thiols in dihydropyrane; synthesis of thioethers

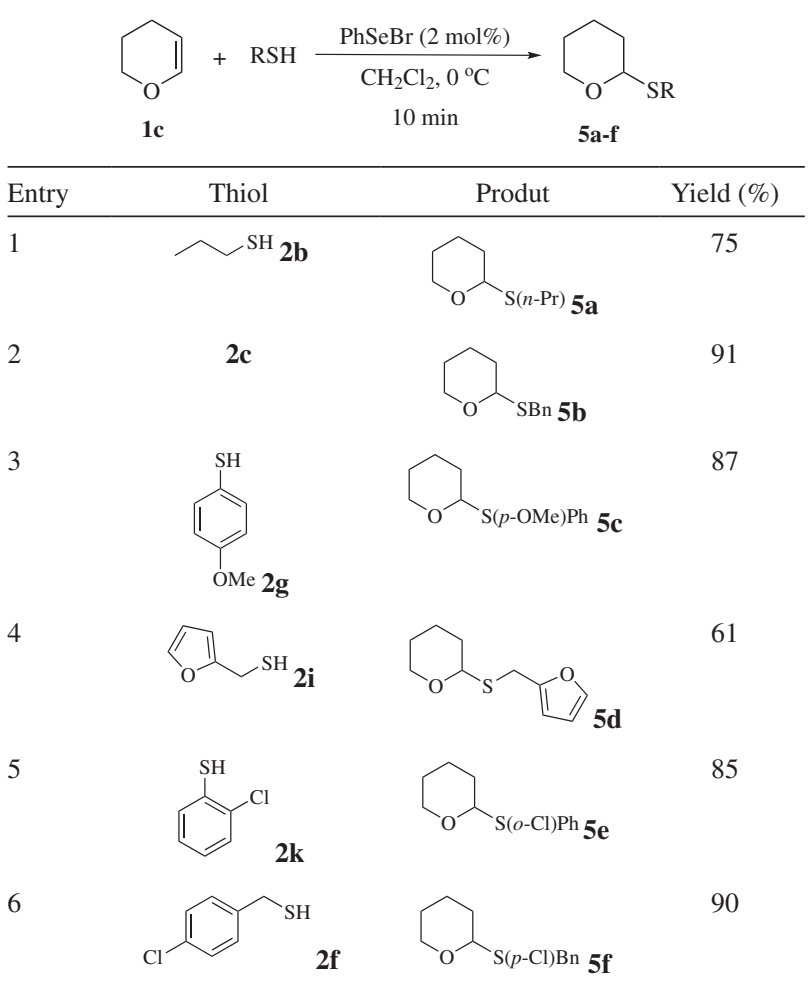

dihydropyrane 1c (1 mmol), benzenethiol $2 \mathrm{a}$ (1.1 mmol), $\mathrm{PhSeBr}(2 \mathrm{~mol} \%)$ and $\mathrm{CH}_{2} \mathrm{Cl}_{2}(1 \mathrm{~mL})$ as a solvent, at $0{ }^{\circ} \mathrm{C}$ for $10 \mathrm{~min}$ gave THP thioether in $75 \%$ yield. The scope and limitations of this protection are summarized in Table 3.

The results showed that the reaction is not sensitive to the electronic effect at aromatic ring in the thiol. For example, both arylthiol bearing electron-donating $(\mathrm{OMe})$ and electron-withdrawing $(\mathrm{Cl})$ group gave the product in good yields. Differentiation in the reactivity between chlorine and sulfur atoms of thiol can also be seen by the reaction of thiols $\mathbf{2 k}$ and $\mathbf{2} \mathbf{f}$ to provide only the THP thioether products in 85 and $90 \%$ yields, respectively, without any side-product observed. In this case, the chlorine substituent was not affected.

\section{Conclusions}

We described herein an efficient method for $\mathrm{PhSeBr}$ mediated addition of thiols to $\alpha, \beta$-unsaturated carbonyl compounds providing a versatile and regioselective synthesis of 1,4-addition products or thioacetals. The reaction was highly controlled by the temperature in which, the 1,4-addition products were obtained when the temperature was $-20^{\circ} \mathrm{C}$; conversely when the reaction was carried out at reflux thioacetals were obtained as a sole product. With this protocol, we were also able to prepare THP thioethers under mild conditions in fair to excellent yields demonstrating the versatility of the $\mathrm{PhSeBr}$ in this catalytic system. The advantages of this method include, the use of cheap, easy and handle catalyst, non anhydrous reaction conditions, non aqueous work-up and ease of product isolation, besides short reaction times and high yields. This reaction associated with the ease in which the protect group can be removed from thiol, can contribute to an interesting alternative route for preparation of more functionalized organothiols.

\section{Supplementary Information}

Experimental details and spectra are available free of charge at http://jbcs.sbq.org.br, as PDF file.

\section{Acknowledgments}

We are grateful to Conselho Nacional de Desenvolvimento Científico e Tecnológico, Coordenação de Aperfeiçoamento de Pessoal de Nível Superior (SAUX) and Fundação de Amparo à Pesquisa do Estado do Rio Grande do Sul, for the fellowships and financial support (PRONEX-10-0005-1). 


\section{References}

1. Greene, T. W.; Wuts, P. G. M.; Protecting Groups in Organic Synthesis, 3rd ed.; John Wiley \& Sons: New York, 1999, pp. 297-348.

2. Cordes, E. H.; Bull, H. G.; Chem. Rev. 1974, 74, 581.

3. Truce, W. E.; Roberts, F. E.; J. Org. Chem. 1963, 28, 961.

4. Garlaschelli, L.; Vidari, G.; Tetrahedron Lett. 1990, 31, 5815.

5. Patney, H. K.; Tetrahedron Lett. 1991, 32, 2259.

6. Ong, B. S.; Tetrahedron Lett. 1980, 21, 4225.

7. Patney, H. K.; Margan, S.; Tetrahedron Lett. 1996, 37, 4621.

8. Tani, H.; Masumoto, K.; Inamasu, T.; Tetrahedron Lett. 1991, 32, 2039.

9. Das, N. B.; Nayak, A.; Sharma, R. P.; J. Chem. Res. (S) 1993, 242.

10. Ku, B.; Oh, D. Y.; Synth. Commun. 1989, 19, 433.

11. Kumar, V.; Dev, S.; Tetrahedron Lett. 1983, 24, 1289.

12. Fieser, L. F.; J. Am. Chem. Soc. 1954, 76, 1945.

13. Khurana, J. M.; Agrawal, A.; Kumar, S.; J. Braz. Chem. Soc. 2009, 20, 1256.

14. Lenardão, E. J.; Trecha, D. O.; Ferreira, P. C.; Jacob, R. G.; Perin, G.; J. Braz. Chem. Soc. 2009, 20, 93.

15. Almeida, Q. A. R.; Pereira, M. L. D. O.; Coelho, R. B.; J. Braz. Chem. Soc. 2008, 19, 894

16. Masaki, Y.; Tanaka, N.; Miura, T.; Tetrahedron Lett. 1998, 39 , 5799.

17. General procedure for $\beta$-mercapto ketones formation: To a Schlenck tube, under air atmosphere containing an appropriate $\alpha, \beta$-unsaturated carbonyl compound $(0.50 \mathrm{mmol})$ in $\mathrm{CH}_{2} \mathrm{Cl}_{2}$ $(2.0 \mathrm{~mL})$, was added the thiol $(0.6 \mathrm{mmol})$. In the resulting solution was added $\mathrm{PhSeBr}(2 \mathrm{~mol} \%)$ and the reaction mixture was allowed to stir for $20 \mathrm{~min}$ at $-20^{\circ} \mathrm{C}$. After that, the mixture was concentrated under vacuum. The residue was purified by flash chromatography on silica gel using ethyl acetate/hexane as the eluent. 3-(Phenylthio)cyclohexanone (3a): Yield 0.162 g (79\%). ${ }^{1} \mathrm{H} \mathrm{NMR}\left(\mathrm{CDCl}_{3}, 400 \mathrm{MHz}\right): \delta$ 7.43-7.40 (m, 2H), 7.33-7.25 (m, 3H), 3.46-3.37 (m, 1H), $2.68(\mathrm{~d}, J 9.7 \mathrm{~Hz}, 1 \mathrm{H})$, 2.40-2.25 (m, 3H), 2.17-2.08 (m, 2H), 1.80-1.63 (m, 2H). ${ }^{13} \mathrm{C}$
NMR ( $\left.\mathrm{CDCl}_{3}, 100 \mathrm{MHz}\right): \delta 208.63,133.09,132.88,128.95$, 127.66, 47.63, 45.98, 40.75, 31.09, 23.90. HRMS calc. for $\mathrm{C}_{12} \mathrm{H}_{24}$ OS: 206.0765. Found: 206.0769.

18. General procedure for thioacetals formation: To a Schlenck tube, under air atmosphere containing an appropriate $\alpha, \beta$-unsaturated carbonyl compounds $(0.50 \mathrm{mmol})$ in $\mathrm{CH}_{2} \mathrm{Cl}_{2}$ $(2.0 \mathrm{~mL})$, was added the thiol $(2.0 \mathrm{mmol})$. In the resulting solution was added $\mathrm{PhSeBr}(2 \mathrm{~mol} \%)$ and the reaction mixture was allowed to stir for $1 \mathrm{~h}$ under reflux. After that, the mixture was concentrated under vacuum. The residue was purified by flash chromatography on silica gel using ethyl acetate/hexane as the eluent. 1,1,3-tris(Phenylthio)cyclohexane (4a): Yield $0.330 \mathrm{~g}(81 \%)$. ${ }^{1} \mathrm{H} \mathrm{NMR}\left(\mathrm{CDCl}_{3}, 200 \mathrm{MHz}\right), \delta: 7.70-7.65$ $(\mathrm{m}, 2 \mathrm{H}), 7.54-7.50(\mathrm{~m}, 2 \mathrm{H}), 7.35-7.20(\mathrm{~m}, 11 \mathrm{H}), 3.56(\mathrm{tt}, J$ 12.0/3.5 Hz, 1H), 2.28-2.19 (m, 1H), 2.03-1.75 (m, 3H), 1.69$1.50(\mathrm{~m}, 3 \mathrm{H}), 1.16-0.94(\mathrm{~m}, 1 \mathrm{H}) .{ }^{13} \mathrm{C} \mathrm{NMR}\left(\mathrm{CDCl}_{3}, 100 \mathrm{MHz}\right)$ $\delta: 137.33,136.11,133.67,132.63,130.90,130.72,129.16$, 128.96, 128.77, 128.61, 128.55, 127.08, 65.05, 43.37, 42.73, 36.12 , 32.15, 22.66. HRMS calc. for $\mathrm{C}_{24} \mathrm{H}_{24} \mathrm{~S}_{3}: 408.1040$. Found: 408.1043.

19. General procedure for thioethers formation: To a Schlenck tube, under air atmosphere containing an appropriate dihydropyrane $(0.50 \mathrm{mmol})$ in $\mathrm{CH}_{2} \mathrm{Cl}_{2}(2.0 \mathrm{~mL})$, was added the thiol $(0.6$ mmol). In the resulting solution was added $\mathrm{PhSeBr}(2 \mathrm{~mol} \%)$ and the reaction mixture was allowed to stir for $20 \mathrm{~min}$ at $0{ }^{\circ} \mathrm{C}$. After this, the mixture was concentrated under vacuum. The residue was purified by flash chromatography on silica gel using ethyl acetate/hexane as the eluent. 2-(Propylthio)-tetrahydro$2 \mathrm{H}$-pyran (5a):Yield $0.120 \mathrm{~g}(75 \%)$. ${ }^{1} \mathrm{H} \mathrm{NMR}\left(\mathrm{CDCl}_{3}, 400\right.$ MHz) $\delta$ : 4.16-3.71 (m, 2H), 3.53-3.36 (m, 1H), 2.72-2.49 (m, 2H), 1.92-1.51 (m, 8H), 0.99 (t, $J 7.20 \mathrm{~Hz}, 3 \mathrm{H}) .{ }^{13} \mathrm{C} \mathrm{NMR}$ $\left(\mathrm{CDCl}_{3}, 100 \mathrm{MHz}\right) \delta: 98.80,67.26,62.26,51.81,35.98,29.21$, $25.44,19.59$. HRMS calc. for $\mathrm{C}_{8} \mathrm{H}_{16} \mathrm{OS}: 160.0921$. Found: 160.0918 . 


\title{
PhSeBr-Catalyzed Selective Addition of Thiols to $\alpha, \beta$-Unsaturated Carbonyl Compounds: Regioselective Synthesis of Thioacetals vs. $\beta$-Mercapto Ketones
}

\author{
Caroline C. Schneider, ${ }^{a}$ Flávia Manarin, ${ }^{a}$ Rodrigo B. Panatieri, ${ }^{b}$ Olga S. R. Barros ${ }^{c}$ and \\ Gilson Zeni ${ }^{*, a}$
}

\author{
${ }^{a}$ Laboratório de Síntese, Reatividade, Avaliação Farmacológica e Toxicidade de \\ Organocalcogênios, CCNE, UFSM, 97105-900 Santa Maria-RS, Brazil \\ ${ }^{b}$ Universidade Federal de Uberlândia, Faculdade de Ciências Integradas do Pontal-Ituiutaba, \\ 38302-000 Ituiutaba-MG, Brazil
}

cInstituto de Química, Universidade Federal de Góias, 74001-970 Goiania-GO, Brazil

Proton nuclear magnetic resonance spectra $\left({ }^{1} \mathrm{H}\right.$ NMR) were obtained at $200 \mathrm{MHz}$ on a DPX-200 NMR spectrometer or at $400 \mathrm{MHz}$ on DPX-400 NMR spectrometer. Spectra were recorded in $\mathrm{CDCl}_{3}$ solutions. Chemical shifts are reported in ppm, referenced to the solvent peak of $\mathrm{CDCl}_{3}$ or tetramethylsilane (TMS) as the external reference. Data are reported as follows: chemical shift $(\delta)$, multiplicity, coupling constant $(J)$ in Hertz and integrated intensity. Carbon-13 nuclear magnetic resonance spectra $\left({ }^{13} \mathrm{C}\right.$ NMR $)$ were obtained either at $50 \mathrm{MHz}$ on a DPX-200 NMR spectrometer or at $100 \mathrm{MHz}$ on a DPX400 NMR spectrometer. Spectra were recorded in $\mathrm{CDCl}_{3}$ solutions. Chemical shifts are reported in ppm, referenced to the solvent peak of $\mathrm{CDCl}_{3}$. Abbreviations to denote the multiplicity of a particular signal are s (singlet), d (doublet), $\mathrm{t}$ (triplet), q (quartet), quint (quintet), sex (sextet) and $\mathrm{m}$ (multiplet). High resolution mass spectra were recorded on a MS50TC double focusing magnetic sector mass spectrometer using EI at $70 \mathrm{eV}$. Column chromatography was performed using Silica Gel (230-400 mesh) following the methods described by Still (Still, W. C.; Kahn, M.; Mitra, A.; J. Org. Chem. 1978, 43, 2923). Thin layer chromatography (TLC) was performed using Silica Gel GF254, $0.25 \mathrm{~mm}$ thickness. For visualization, TLC plates were either placed under ultraviolet light, or stained with iodine vapour, or acidic vanillin. Most reactions were monitored by TLC for disappearance of starting material. Air- and moisture-sensitive reactions were conducted in flame-dried or oven dried glassware equipped with tightly fitted rubber septa and under a positive atmosphere of dry argon. Reagents and solvents were handled using standard

*e-mail: gzeni@pq.cnpq.br syringe techniques. Temperatures above room temperature were maintained by use of a mineral oil bath with an electrically heated coil connected to a Variac controller.

General procedure for $\beta$-mercapto ketones

To a Schlenck tube, under air atmosphere containing an appropriate $\alpha, \beta$-unsaturated carbonyl compounds $(0.50 \mathrm{mmol})$ in $\mathrm{CH}_{2} \mathrm{Cl}_{2}(2.0 \mathrm{~mL})$, was added the thiol $(0.6 \mathrm{mmol})$. In the resulting solution was added $\mathrm{PhSeBr}$ ( $2 \mathrm{~mol} \%)$ and the reaction mixture was allowed to stir for $20 \mathrm{~min}$ at $-20{ }^{\circ} \mathrm{C}$. After that, the mixture was concentrated under vacuum. The residue was purified by flash chromatography on silica gel using ethyl acetate/ hexane as the eluent.

\section{3-(Phenylthio)cyclohexanone (3a)}

Yield: $0.162 \mathrm{~g}(79 \%) .{ }^{1} \mathrm{H}$ NMR $\left(\mathrm{CDCl}_{3}, 400 \mathrm{MHz}\right)$ $\delta: ~ 7.43-7.40(\mathrm{~m}, 2 \mathrm{H}), 7.33-7.25(\mathrm{~m}, 3 \mathrm{H}), 3.46-3.37(\mathrm{~m}$, $1 \mathrm{H}), 2.68(\mathrm{~d}, J 9.7 \mathrm{~Hz}, 1 \mathrm{H}), 2.40-2.25(\mathrm{~m}, 3 \mathrm{H}), 2.17-2.08$

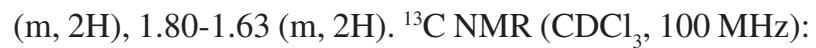
$\delta$ 208.63, 133.09, 132.88, 128.95, 127.66, 47.63, 45.98, 40.75, 31.09, 23.90. HRMS calc. for $\mathrm{C}_{12} \mathrm{H}_{24}$ OS: 206.0765 . Found: 206.0769 .

\section{3-(Propylthio)cyclohexanone (3b)}

Yield: $0.127 \mathrm{~g}(74 \%) .{ }^{1} \mathrm{H}$ NMR $\left(\mathrm{CDCl}_{3}, 400 \mathrm{MHz}\right)$ $\delta: 3.08-3.01(\mathrm{~m}, 1 \mathrm{H}), 2.70(\mathrm{~d}, J 9.5 \mathrm{~Hz}, 1 \mathrm{H}), 2.53(\mathrm{t}, J 7.4$ $\mathrm{Hz}, 2 \mathrm{H}), 2.41-2.31$ (m, 3H), 2.18-2.10 (m, 2H), 1.76-1.68 (m, 2H), 1.60 (sex, J 7.3 Hz, 2H), 0.99 (t, J 7.3 Hz, 3H). ${ }^{13} \mathrm{C}$ NMR $\left(\mathrm{CDCl}_{3}, 100 \mathrm{MHz}\right) \delta: 208.61,48.00,42.49$, 40.70, 32.31, 31.42, 23.98, 22.82, 13.27. HRMS calc. for $\mathrm{C}_{9} \mathrm{H}_{16}$ OS: 172.0921. Found: 172.0927. 


\section{3-(Benzylthio) cyclohexanone (3c)}

Yield: $0.138 \mathrm{~g}(63 \%) .{ }^{1} \mathrm{H} \mathrm{NMR}\left(\mathrm{CDCl}_{3}, 200 \mathrm{MHz}\right)$, $\delta: 7.32-7.21(\mathrm{~m}, 5 \mathrm{H}), 3.76(\mathrm{~s}, 2 \mathrm{H}), 3.00-2.86(\mathrm{~m}, 1 \mathrm{H})$, 2.67 (dd, $J 9.6 / 4.4 \mathrm{~Hz}, 1 \mathrm{H}), 2.40-2.29(\mathrm{~m}, 3 \mathrm{H}), 2.17-2.02$ (m, 2H), 1.73-1.61 (m, 2H). ${ }^{13} \mathrm{C} \mathrm{NMR}\left(\mathrm{CDCl}_{3}, 100 \mathrm{MHz}\right)$ $\delta: 208.13,137.61,128.39,128.23,126.75,47.39,41.67$, 40.55, 34.55, 30.86, 23.70. MS (relative intensity) $m / z: 220$ (11), 123 (32), 97 (53), 91 (100). HRMS calc. for $\mathrm{C}_{13} \mathrm{H}_{16} \mathrm{OS}$ : 220.0921. Found: 220.0925.

\section{3-(terc-Butylthio)cyclohexanone (3d)}

Yield: $0.137 \mathrm{~g}(74 \%) .{ }^{1} \mathrm{H}$ NMR $\left(\mathrm{CDCl}_{3}, 400 \mathrm{MHz}\right)$ $\delta: 3.03-2.96(\mathrm{~m}, 1 \mathrm{H}), 2.72(\mathrm{dd}, J 9.3 / 4.7 \mathrm{~Hz}, 1 \mathrm{H}), 2.43-2.22$ (m, 3H), 2.20-2.06 (m, 2H), 1.77-1.69 (m, 2H), $1.34(\mathrm{~s}$, 9H). ${ }^{13} \mathrm{C} \mathrm{NMR}\left(\mathrm{CDCl}_{3}, 100 \mathrm{MHz}\right) \delta: 208.92,50.66,43.49$, 40.51, 40.46, 34.13, 31.27, 24.46. MS (relative intensity) m/z: 186 (30), 171 (1), 130 (29), 96 (50). HRMS calc. for $\mathrm{C}_{10} \mathrm{H}_{18} \mathrm{OS}$ : 186.1078. Found: 186.1081 .

\section{3-(Dodecylthio) cyclohexanone (3e)}

Yield: $0.226 \mathrm{~g}(76 \%) .{ }^{1} \mathrm{H} \mathrm{NMR}\left(\mathrm{CDCl}_{3}, 400 \mathrm{MHz}\right)$ $\delta: 3.09-3.01(\mathrm{~m}, 1 \mathrm{H}), 2.71(\mathrm{dd}, J 9.6 / 4.3 \mathrm{~Hz}, 1 \mathrm{H}), 2.54(\mathrm{t}$, $J 7.5 \mathrm{~Hz}, 2 \mathrm{H}), 2.40-2.31(\mathrm{~m}, 3 \mathrm{H}), 2.17-2.10(\mathrm{~m}, 2 \mathrm{H}), 1.77-$ $1.67(\mathrm{~m}, 2 \mathrm{H}), 1.59-1.53(\mathrm{~m}, 3 \mathrm{H}), 1.40-1.26(\mathrm{~m}, 17 \mathrm{H}), 0.88$ (t, $J 7.5 \mathrm{~Hz}, 3 \mathrm{H}) .{ }^{13} \mathrm{C} \mathrm{NMR}\left(\mathrm{CDCl}_{3}, 100 \mathrm{MHz}\right) \delta: 199.60$, $39.05,37.92,31.78,29.50,29.47,29.39,29.22,29.11$, $29.09,28.39,25.56,22.55,13.98$. MS (relative intensity) m/z: 267 (1), 201 (45), 129 (4), 97 (22). HRMS calc. for $\mathrm{C}_{18} \mathrm{H}_{34}$ OS: 298.2330. Found: 298.2332.

\section{3-(sec-Butylthio)cyclohexanone (3f)}

Yield: $0.113 \mathrm{~g}(61 \%) .{ }^{1} \mathrm{H} \mathrm{NMR}\left(\mathrm{CDCl}_{3}, 400 \mathrm{MHz}\right)$ $\delta: 3.14-3.04$ (m, 1H), 2.77 (sex d, J 7.0/2.3 Hz, 1H), 2.69 (dd, $J 9.5 / 4.2,1 \mathrm{H}), 2.40-2.31(\mathrm{~m}, 3 \mathrm{H}), 2.18-2.09(\mathrm{~m}, 2 \mathrm{H})$, $1.78-1.67(\mathrm{~m}, 2 \mathrm{H}), 1.63-1.45(\mathrm{~m}, 2 \mathrm{H}), 1.25(\mathrm{~d}, J 6.7 \mathrm{~Hz}$, $3 \mathrm{H}), 0.97(\mathrm{t}, J 7.4 \mathrm{~Hz}, 3 \mathrm{H}) \cdot{ }^{13} \mathrm{C} \mathrm{NMR}\left(\mathrm{CDCl}_{3}, 100 \mathrm{MHz}\right)$ $\delta: 208.79,48.54,41.34,40.78,40.40,31.89,29.79,24.09$, 20.98, 11.10. MS (relative intensity) $\mathrm{m} / z$ : $186(30), 171$ (1), 157 (20), 143 (1), 129 (21), 97 (100). HRMS calc. for $\mathrm{C}_{10} \mathrm{H}_{18} \mathrm{OS}: 186.1078$. Found: 186.1083 .

\section{3-(4-Methoxyphenylthio)cyclohexanone (3g)}

Yield: $0.224 \mathrm{~g}(95 \%) .{ }^{1} \mathrm{H} \mathrm{NMR}\left(\mathrm{CDCl}_{3}, 400 \mathrm{MHz}\right)$ $\delta$ : 7.41-7.37 (m, 2H), 6.86-6.78 (m, 2H), $3.80(\mathrm{~s}, 3 \mathrm{H})$, 3.27-3.19 (m, 1H), $2.61(\mathrm{dd}, J 9.6 / 4.4 \mathrm{~Hz}, 1 \mathrm{H}), 2.35-2.26$ (m, 3H), 2.16-2.04 (m, 2H), 1.73-1.64 (m, 2H). ${ }^{13} \mathrm{C} \mathrm{NMR}$ $\left(\mathrm{CDCl}_{3}, 100 \mathrm{MHz}\right) \delta: 208.61,159.70,136.17,122.70$, $114.33,55.05,47.45,46.72,40.55,30.91,23.73$. MS (relative intensity) $m / z: 236$ (70), 207 (1), 140 (100), 97 (19). HRMS calc. for $\mathrm{C}_{13} \mathrm{H}_{16} \mathrm{O}_{2} \mathrm{~S}: 236.0871$. Found: 236.0867.

\section{3-(4-Chlorophenylthio)cyclohexanone (3h)}

Yield: $0.180 \mathrm{~g}(75 \%) .{ }^{1} \mathrm{H} \mathrm{NMR}\left(\mathrm{CDCl}_{3}, 400 \mathrm{MHz}\right)$ $\delta: ~ 7.37-7.33(\mathrm{~m}, 2 \mathrm{H}), 7.30-7.26(\mathrm{~m}, 2 \mathrm{H}), 3.43-3.36(\mathrm{~m}$, $1 \mathrm{H}), 2.66(\mathrm{dd}, J 9.4 / 4.5 \mathrm{~Hz}, 1 \mathrm{H}), 2.39-2.26(\mathrm{~m}, 3 \mathrm{H}), 2.18-$ $2.09(\mathrm{~m}, 2 \mathrm{H}), 1.77-1.67(\mathrm{~m}, 2 \mathrm{H}) .{ }^{13} \mathrm{C} \mathrm{NMR}\left(\mathrm{CDCl}_{3}, 100\right.$ MHz) $\delta: 208.28,134.48,133.99,131.45,129.17,47.52$, 46.27, 40.74, 31.05, 23.87. HRMS calc. for $\mathrm{C}_{12} \mathrm{H}_{13}$ ClOS: 240.0375. Found: 240.0379 .

\section{3-(Furan-2-ylmethylthio)cyclohexanone (3i)}

Yield: $0.176 \mathrm{~g}(84 \%) .{ }^{1} \mathrm{H} \mathrm{NMR}\left(\mathrm{CDCl}_{3}, 200 \mathrm{MHz}\right)$ $\delta: 7.35(\mathrm{dd}, J 1.7 / 0.7 \mathrm{~Hz}, 1 \mathrm{H}), 7.30(\mathrm{dd}, J 3.1 / 1.9 \mathrm{~Hz}, 1 \mathrm{H})$, 6.18 (dd, $J 3.1 / 0.7 \mathrm{~Hz}, 1 \mathrm{H}), 3.77$ (s, 2H), 3.08-3.00 (m, $1 \mathrm{H}), 2.69$ (dd, J 9.3/4.2 Hz, 1H), 2.40-2.30 (m, 3H), 2.152.08 (m, 2H), 1.76-1.65 (m, 2H). ${ }^{13} \mathrm{C} \mathrm{NMR}\left(\mathrm{CDCl}_{3}, 100\right.$ MHz) $\delta:$ 208.61, 151.24, 142.10, 110.42, 107.40, 47.65, $42.32,40.82,31.14,26.94,24.05$. MS (relative intensity) $m / z: 210$ (6), 114 (20), 97 (12), 81 (100). HRMS calc. for $\mathrm{C}_{11} \mathrm{H}_{14} \mathrm{O}_{2} \mathrm{~S}: 210.0714$. Found: 210.0719 .

\section{3-(2-Chlorophenylthio)cyclohexanone (3k)}

Yield: $0.170 \mathrm{~g}(71 \%) .{ }^{1} \mathrm{H} \mathrm{NMR}\left(\mathrm{CDCl}_{3}, 200 \mathrm{MHz}\right)$ $\delta: 7.45-7.39(\mathrm{~m}, 2 \mathrm{H}), 7.25-7.18(\mathrm{~m}, 2 \mathrm{H}), 3.69-3.52(\mathrm{~m}, 1 \mathrm{H})$, 2.69 (dd, $J$ 9.4/4.4 Hz, 1H), 2.48-2.32 (m, 3H), 2.23-2.07 (m, 2H), 1.87-1.66 (m, 2H). ${ }^{13} \mathrm{C} \mathrm{NMR}\left(\mathrm{CDCl}_{3}, 50 \mathrm{MHz}\right)$ $\delta: 208.19,136.79,133.61,132.41,130.07,128.64,127.13$, 47.41, 44.74, 40.80, 30.91, 23.95. MS (relative intensity) m/z: 240 (47), 205 (1), 108 (20), 97 (80). HRMS calc. for $\mathrm{C}_{12} \mathrm{H}_{13}$ ClOS: 240.0375. Found: 240.0380 .

3-(Furan-2-ylmethylthio)-4,4-dimethylcyclohexanone (3l)

Yield: $0.185 \mathrm{~g}(78 \%) .{ }^{1} \mathrm{H} \mathrm{NMR}\left(\mathrm{CDCl}_{3}, 200 \mathrm{MHz}\right)$ $\delta: 7.35(\mathrm{~d}, J 0.9 \mathrm{~Hz}, 1 \mathrm{H}), 6.30(\mathrm{dd}, J 3.0 / 1.8 \mathrm{~Hz}, 1 \mathrm{H}), 6.17$ $(\mathrm{d}, J 3.0 \mathrm{~Hz}, 1 \mathrm{H}), 3.73(\mathrm{~s}, 2 \mathrm{H}), 2.73-4.49(\mathrm{~m}, 3 \mathrm{H}), 2.44-2.21$ (m, 2H), 1.88-1.77 (m, 1H), 1.65-1.49 (m, 1H), $1.10(\mathrm{~s}, 6 \mathrm{H})$. ${ }^{13} \mathrm{C}$ NMR $\left(\mathrm{CDCl}_{3}, 100 \mathrm{MHz}\right) \delta: 209.12,151.25,142.15$, $110.37,107.65,53.22,45.59,38.67,37.78,34.60,28.39$, 25.21, 20.56. MS (relative intensity) $\mathrm{m} / z: 238$ (10), 125 (23), 97 (3), 81 (100), 69 (14). HRMS calc. for $\mathrm{C}_{13} \mathrm{H}_{18} \mathrm{O}_{2} \mathrm{~S}$ : 238.1027. Found: 238.1032 .

\section{4,4-Dimethyl-3-(phenylthio)cyclohexanone (3m)}

Yield: $0.145 \mathrm{~g}(62 \%) .{ }^{1} \mathrm{H} \mathrm{NMR}\left(\mathrm{CDCl}_{3}, 200 \mathrm{MHz}\right)$ $\delta: 7.41-7.38(\mathrm{~m}, 2 \mathrm{H}), 7.31-7.22(\mathrm{~m}, 3 \mathrm{H}), 3.19-3.15(\mathrm{~m}, 1 \mathrm{H})$, 2.66-2.52 (m, 2H), 2.50-2.41 (m, 1H), 2.34-2.27 (m, 1H), $1.93-1.87(\mathrm{~m}, 1 \mathrm{H}), 1.68,1.59(\mathrm{~m}, 1 \mathrm{H}), 1.28(\mathrm{~s}, 3 \mathrm{H}), 1.22$ (s, 3H). ${ }^{13} \mathrm{C}$ NMR $\left(\mathrm{CDCl}_{3}, 100 \mathrm{MHz}\right) \delta: 208.93,134.57$, $132.64,129.05,127.36,57.58,45.37,38.58,37.82,34.60$, $28.99,20.97$. HRMS calc. for $\mathrm{C}_{14} \mathrm{H}_{18} \mathrm{OS}: 234.1078$. Found: 234.1083. 


\section{General procedure for thioacetals formation}

To a Schlenck tube, under air atmosphere containing an appropriate $\alpha, \beta$-unsaturated carbonyl compounds $(0.50$ $\mathrm{mmol})$ in $\mathrm{CH}_{2} \mathrm{Cl}_{2}(2.0 \mathrm{~mL})$, was added the thiol $(2.0 \mathrm{mmol})$. In the resulting solution was added $\mathrm{PhSeBr}(2 \mathrm{~mol} \%)$ and the reaction mixture was allowed to stir for $1 \mathrm{~h}$ under reflux. After that, the mixture was concentrated under vacuum. The residue was purified by flash chromatography on silica gel using ethyl acetate/hexane as the eluent.

\section{1,1,3-tris(Phenylthio)cyclohexane (4a)}

Yield: $0.330 \mathrm{~g}(81 \%) .{ }^{1} \mathrm{H} \mathrm{NMR}\left(\mathrm{CDCl}_{3}, 200 \mathrm{MHz}\right)$ $\delta$ : 7.70-7.65 (m, 2H), 7.54-7.50 (m, 2H), 7.35-7.20 (m, $11 \mathrm{H}), 3.56(\mathrm{tt}, J 12.0 / 3.5 \mathrm{~Hz}, 1 \mathrm{H}), 2.28-2.19(\mathrm{~m}, 1 \mathrm{H})$, 2.03-1.75 (m, 3H), 1.69-1.50 (m, 3H), 1.16-0.94 (m, 1H). ${ }^{13} \mathrm{C}$ NMR $\left(\mathrm{CDCl}_{3}, 100 \mathrm{MHz}\right) \delta: 137.33,136.11,133.67$, $132.63,130.90,130.72,129.16,128.96,128.77,128.61$, 128.55, 127.08, 65.05, 43.37, 42.73, 36.12, 32.15, 22.66. HRMS calc. for $\mathrm{C}_{24} \mathrm{H}_{24} \mathrm{~S}_{3}: 408.1040$. Found: 408.1043.

\section{1,1,3-tris(Propylthio)cyclohexane (4b)}

Yield: $0.253 \mathrm{~g}(83 \%) .{ }^{1} \mathrm{H} \mathrm{NMR}\left(\mathrm{CDCl}_{3}, 200 \mathrm{MHz}\right)$ $\delta: 3.06(\mathrm{tt}, J 11.8 / 3.9 \mathrm{~Hz}, 1 \mathrm{H}), 2.63(\mathrm{t}, J 7.0 \mathrm{~Hz}, 2 \mathrm{H}), 2.51$ (t, J 7.0 Hz, 4H), 2.33-2.19 (m, 1H), 2.09-1.81 (m, 2H), $1.78-1.72(\mathrm{~m}, 2 \mathrm{H}), 1.69-1.50(\mathrm{~m}, 8 \mathrm{H}), 1.31-1.11(\mathrm{~m}, 1 \mathrm{H})$, $1.00(\mathrm{t}, J 7.0,3 \mathrm{H}), 0.99(\mathrm{t}, J 7.0,3 \mathrm{H}), 0.98(\mathrm{t}, J 7.0,3 \mathrm{H})$. ${ }^{13} \mathrm{C} \mathrm{NMR}\left(\mathrm{CDCl}_{3}, 100 \mathrm{MHz}\right) \delta: 61.14,44.87,39.02,36.94$, $33.13,32.03,30.56,30.17,23.16,22.65,22.48,22.32$, 13.73, 13.67, 13.36. MS (relative intensity) $\mathrm{m} / \mathrm{z}: 306(8)$, 263 (1), 231 (67), 155 (100). HRMS calc. for $\mathrm{C}_{15} \mathrm{H}_{30} \mathrm{~S}_{3}$ : 306.1509. Found: 306.1513 .

\section{1,1,3-tris(Benzylthio)cyclohexane (4c)}

Yield: $0.427 \mathrm{~g}(95 \%) .{ }^{1} \mathrm{H} \mathrm{NMR}\left(\mathrm{CDCl}_{3}, 200 \mathrm{MHz}\right)$ $\delta: 7.35-7.23(\mathrm{~m}, 15 \mathrm{H}), 3.88$ (s, 2H), 3.71 (d, J 2.5 Hz, 2H), 3.69 (s, 2H), 3.02 (tt, J 12.1/3.9 Hz, 1H), 2.30-2.18 (m, 1H), 2.04-1.90 (m, 2H), 1.81-1.57 (m, 4H), 1.32-1.28 (m, 1H). ${ }^{13} \mathrm{C}$ NMR $\left(\mathrm{CDCl}_{3}, 100 \mathrm{MHz}\right) \delta: 138.33,137.68,137.27$, $128.91,128.50,128.24,126.72,126.65,62.73,44.31$, $38.98,36.68,34.65,33.39,33.21,32.60,22.34$. HRMS calc. for $\mathrm{C}_{27} \mathrm{H}_{30} \mathrm{~S}_{3}: 450.1509$. Found: 450.1505 .

\section{1,1,3-tris(Dodecylthio)cyclohexane (4e)}

Yield: $0.601 \mathrm{~g}(88 \%) .{ }^{1} \mathrm{H} \mathrm{NMR}\left(\mathrm{CDCl}_{3}, 200 \mathrm{MHz}\right)$ $\delta: 3.06(\mathrm{tt}, J 12.0 / 3.3 \mathrm{~Hz}, 1 \mathrm{H}), 2.64(\mathrm{t}, J 7.3 \mathrm{~Hz}, 2 \mathrm{H})$, 2.57-2.46 (m, 4H), 2.30-2.20 (m, 1H), 2.08-1.81 (m, 2H), $1.78-1.66$ (m, 3H), 1.64-1.49 (m, 6H), 1.40-1.19 (m, 56H), $0.88(\mathrm{t}, J 6.7 \mathrm{~Hz}, 9 \mathrm{H}) .{ }^{13} \mathrm{C} \mathrm{NMR}\left(\mathrm{CDCl}_{3}, 100 \mathrm{MHz}\right) \delta$ : 61.20, 44.79, 39.04, 38.91, 37.10, 33.99, 33.20, 31.80, $30.00,29.93,29.54,29.45,29.25,29.20,28.99,28.87$,
28.51, 28.28, 28.09, 24.35, 22.54, 13.90. HRMS calc. for $\mathrm{C}_{42} \mathrm{H}_{84} \mathrm{~S}_{3}: 684.5735$. Found: 684.5740 .

\section{1,1,3-tris(sec-Butylthio)cyclohexane (4f)}

Yield: $0.208 \mathrm{~g}(60 \%) .{ }^{1} \mathrm{H} \mathrm{NMR}\left(\mathrm{CDCl}_{3}, 400 \mathrm{MHz}\right)$ $\delta: 3.18-3.08(\mathrm{~m}, 1 \mathrm{H}), 3.03-2.96(\mathrm{~m}, 2 \mathrm{H}), 2.81-2.73(\mathrm{~m}$, $1 \mathrm{H}), 2.32-2.23(\mathrm{~m}, 1 \mathrm{H}), 2.05-1.91(\mathrm{~m}, 3 \mathrm{H}), 1.79-1.42(\mathrm{~m}$, 10H), 1.31-1.21 (m, 9H), 0.96 (t, J 7.3 Hz, 9H). ${ }^{13} \mathrm{C}$ NMR $\left(\mathrm{CDCl}_{3}, 100 \mathrm{MHz}\right) \delta: 62.82,46.26,40.02,39.21,38.07$, $33.65,33.61,31.62,31.47,31.27,30.08,29.95,22.67$, 22.08, 21.43, 11.21, 11.13, 11.01. MS (relative intensity) m/z: 348 (3), 259 (80), 169 (100), 113 (93). HRMS calc. for $\mathrm{C}_{18} \mathrm{H}_{36} \mathrm{~S}_{3}: 348.1979$. Found: 348.1982 .

\section{1,1,3-tris(4-Methoxyphenylthio)cyclohexane (4g)}

Yield: $0.428 \mathrm{~g}(86 \%) .{ }^{1} \mathrm{H} \mathrm{NMR}\left(\mathrm{CDCl}_{3}, 400 \mathrm{MHz}\right)$ $\delta: 7.49$ (d, J 9.0 Hz, 2H), 7.39 (d, J $8.5 \mathrm{~Hz}, 2 \mathrm{H}), 7.29$ (d, $J 8.8 \mathrm{~Hz}, 2 \mathrm{H}), 6.82-6.77(\mathrm{~m}, 6 \mathrm{H}), 3.78$ (s, 9H), 3.36 (tt, $J$ 11.9/3.4 Hz, 1H), 2.07-1.93 (m, 2H), 1.92-1.70 (m, 2H), 1.58-1.49 (m, 3H), 1.04-0.95 (m, 1H). ${ }^{13} \mathrm{C} \mathrm{NMR}\left(\mathrm{CDCl}_{3}\right.$, $100 \mathrm{MHz}) \delta: 160.40,160.28,159.50,138.83,137.99$, 136.27, 123.39, 121.84, 121.09, 114.19, 113.97, 64.69, 55.08, 43.75, 42.77, 35.72, 31.98, 22.62. MS (relative intensity) m/z: 359 (38), 219 (100), 187 (27), 139 (49), 77 (13). HRMS calc. for $\mathrm{C}_{27} \mathrm{H}_{30} \mathrm{O}_{3} \mathrm{~S}_{3}: 498.1357$. Found: 498.1355 .

2-((1,3-bis(Furan-2-ylmethylthio)cyclohexylthio)methyl) furan (4h)

Yield: $0.344 \mathrm{~g}(82 \%) .{ }^{1} \mathrm{H} \mathrm{NMR}\left(\mathrm{CDCl}_{3}, 400 \mathrm{MHz}\right)$ $\delta: 7.38-7.32(\mathrm{~m}, 3 \mathrm{H}), 6.30-6.29(\mathrm{~m}, 3 \mathrm{H}), 6.20-6.17(\mathrm{~m}, 3 \mathrm{H})$, $3.94(\mathrm{~s}, 2 \mathrm{H}), 3.83(\mathrm{~s}, 2 \mathrm{H}), 3.70(\mathrm{~s}, 2 \mathrm{H}), 3.05(\mathrm{tt}, J$ 12.2/3.8 $\mathrm{Hz}, 1 \mathrm{H}), 2.27-2.22$ (m, 1H), 2.02-1.92 (m, 2H), 1.82-1.56 (m, 4H), 1.20 (qd, $J 8.4 / 4.0 \mathrm{~Hz}, 1 \mathrm{H}) .{ }^{13} \mathrm{C} \mathrm{NMR}\left(\mathrm{CDCl}_{3}, 100\right.$ MHz) $\delta: 151.64,151.29,150.93,141.75,141.69,110.35$, 110.28, 107.34, 107.27, 107.04, 62.76, 44.14, 39.23, 36.55, $32.43,26.74,25.70,25.47,22.32$. MS (relative intensity) m/z: 339 (15), 306 (1), 225 (10), 81 (100). HRMS calc. for $\mathrm{C}_{21} \mathrm{H}_{24} \mathrm{O}_{3} \mathrm{~S}_{3}: 420.0887$. Found: 420.0890 .

2-((1,3-bis(Furan-2-ylmethylthio)-4,4-dimethylcyclohexylthio)methyl)furan (4k)

Yield: $0.340 \mathrm{~g}(76 \%) .{ }^{1} \mathrm{H} \mathrm{NMR}\left(\mathrm{CDCl}_{3}, 400 \mathrm{MHz}\right)$ $\delta:$ 7.35-7.33 (m, 3H), 6.31-6.28 (m, 3H), 6.19-6.18 (m, $3 \mathrm{H}), 3.92$ (s, 2H), 3.82 (d, J $1.7 \mathrm{~Hz}, 2 \mathrm{H}), 3.64$ (d, J 3.2 $\mathrm{Hz}, 2 \mathrm{H}), 2.83$ (t, J 8.1 Hz, 1H), 2.04 (d, J $9.3 \mathrm{~Hz}, 2 \mathrm{H})$, 1.93-1.84 (m, 1H), 1.81-1.73 (m, 2H), 1.29-1.24 (m, $1 \mathrm{H}), 0.99$ (s, 3H), 0.85 (s, 3H). ${ }^{13} \mathrm{C} \mathrm{NMR}\left(\mathrm{CDCl}_{3}, 100\right.$ $\mathrm{MHz}) \delta: 151.62,151.34,151.06,141.88,141.82,110.47$, 110.43, 110.33, 107.51, 107.49, 107.38, 63.11, 51.06, $41.85,36.68,34.50,33.00,29.74,28.80,25.87,25.60$, 
19.74. MS (relative intensity) $m / z: 367$ (9), 334 (7), 253 (11), 221 (6), 81 (100). HRMS calc. for $\mathrm{C}_{23} \mathrm{H}_{28} \mathrm{O}_{3} \mathrm{~S}_{3}$ : 448.1200. Found: 448.1205.

\section{1-((5,5-bis(Benzylthio)-2,2-dimethylcyclohexylthio)methyl)} benzene $(4 \mathrm{l})$

Yield: $0.425 \mathrm{~g}(89 \%) .{ }^{1} \mathrm{H} N M R\left(\mathrm{CDCl}_{3}, 200 \mathrm{MHz}\right) \delta$ : 7.35-7.16 (m, 15H), $3.86(\mathrm{~s}, 2 \mathrm{H}), 3.68(\mathrm{~d}, J 3.5 \mathrm{~Hz}, 2 \mathrm{H})$, $3.58(\mathrm{~s}, 2 \mathrm{H}), 2.85-2.76(\mathrm{~m}, 1 \mathrm{H}), 2.08-2.03(\mathrm{~m}, 2 \mathrm{H}), 1.92-$ $1.69(\mathrm{~m}, 3 \mathrm{H}), 1.31-1.22(\mathrm{~m}, 1 \mathrm{H}), 1.00(\mathrm{~s}, 1 \mathrm{H}), 0.86(\mathrm{~s}, 3 \mathrm{H})$. ${ }^{13} \mathrm{C}$ NMR $\left(\mathrm{CDCl}_{3}, 100 \mathrm{MHz}\right) \delta: 138.50,137.70,137.42$, $129.02,128.99,128.78,128.33,128.25,126.80,126.74$, 63.08, 50.61, 41.88, 36.69, 34.45, 33.46, 32.97, 29.93, 19.72 . HRMS calc. for $\mathrm{C}_{29} \mathrm{H}_{34} \mathrm{~S}_{3}: 478.1822$. Found: 478.1819 .

1-(1,3-bis(4-Methoxyphenylthio)-4,4-dimethylcyclohexylthio)-4-methoxybenzene (4m)

Yield: $0.483 \mathrm{~g}(92 \%) .{ }^{1} \mathrm{H} \mathrm{NMR}\left(\mathrm{CDCl}_{3}, 400 \mathrm{MHz}\right)$ $\delta: ~ 7.45-7.43(\mathrm{~m}, 2 \mathrm{H}), 7.34-7.30(\mathrm{~m}, 4 \mathrm{H}), 6.86-6.81(\mathrm{~m}$, $4 \mathrm{H}), 6.69-6.67(\mathrm{~m}, 2 \mathrm{H}), 3.83(\mathrm{~s}, 3 \mathrm{H}), 3.81(\mathrm{~s}, 3 \mathrm{H}), 3.79$ (s, 3H), 3.24-3.20 (m, 1H), 1.96-1.85 (m, 4H), 1.60-1.53 $(\mathrm{m}, 1 \mathrm{H}), 1.28-1.25(\mathrm{~m}, 1 \mathrm{H}), 1.21(\mathrm{~s}, 3 \mathrm{H}), 0.67(\mathrm{~s}, 3 \mathrm{H})$ ${ }^{13} \mathrm{C}$ NMR $\left(\mathrm{CDCl}_{3}, 100 \mathrm{MHz}\right) \delta: 160.44,160.21,159.30$, $138.83,138.16,135.79,132.55,125.77,121.02,114.42$, $114.05,113.94,64.60,56.89,55.19,55.07,39.64,37.33$, $34.21,32.21,30.47,19.45$. MS (relative intensity) $\mathrm{m} / \mathrm{z}: 416$ (1), 387 (34), 247 (100), 139 (49), 107 (50). HRMS calc. for $\mathrm{C}_{29} \mathrm{H}_{34} \mathrm{O}_{3} \mathrm{~S}_{3}: 526.1670$. Found: 526.1673 .

\section{1,1-Dimethyl-2,4,4-tris(propylthio)cyclohexane (4n)}

Yield: $0.260 \mathrm{~g}(78 \%) .{ }^{1} \mathrm{H} \mathrm{NMR}\left(\mathrm{CDCl}_{3}, 400 \mathrm{MHz}\right)$ $\delta: 2.81(\mathrm{dd}, J 9.5 / 4.4 \mathrm{~Hz}, 1 \mathrm{H}), 2.64(\mathrm{t}, J 7.4 \mathrm{~Hz}, 2 \mathrm{H}), 2.48$ (t, $J 7.4 \mathrm{~Hz}, 4 \mathrm{H}), 2.20-1.72$ (m, 5H), 1.60 (sex, $J 7.4 \mathrm{~Hz}$, $6 \mathrm{H}), 1.40-1.20(\mathrm{~m}, 1 \mathrm{H}), 1.11(\mathrm{~s}, 3 \mathrm{H}), 1.00(\mathrm{t}, J 7.4 \mathrm{~Hz}$, $3 \mathrm{H}), 1.09$ (t, J 7.4 Hz, 3H), 0.98 (t, J 7.4 Hz, 3H), 0.88 (s, $3 \mathrm{H}) .{ }^{13} \mathrm{C} \mathrm{NMR}\left(\mathrm{CDCl}_{3}, 100 \mathrm{MHz}\right) \delta: 61.39,51.05,42.46$, $36.88,34.55,33.25,30.68,30.29,30.19,23.15,22.68$, $22.38,19.54,13.79,13.74,13.30$. HRMS calc. for $\mathrm{C}_{17} \mathrm{H}_{34} \mathrm{~S}_{3}$ : 334.1822. Found: 334.1820.

\section{General procedure for thioethers formation}

To a Schlenck tube, under air atmosphere containing an appropriate dihydropyrane $(0.50 \mathrm{mmol})$ in $\mathrm{CH}_{2} \mathrm{Cl}_{2}$ $(2.0 \mathrm{~mL})$, was added the thiol $(0.6 \mathrm{mmol})$. In the resulting solution was added $\mathrm{PhSeBr}(2 \mathrm{~mol} \%)$ and the reaction mixture was allowed to stir for $20 \mathrm{~min}$ at $0{ }^{\circ} \mathrm{C}$ for thioethers products. After that, the mixture was concentrated under vacuum. The residue was purified by flash chromatography on silica gel using ethyl acetate/ hexane as the eluent.

\section{2-(Propylthio)-tetrahydro-2H-pyran (5a)}

Yield: $0.120 \mathrm{~g}(75 \%) .{ }^{1} \mathrm{H}$ NMR $\left(\mathrm{CDCl}_{3}, 400 \mathrm{MHz}\right)$ $\delta: 4.16-3.71(\mathrm{~m}, 2 \mathrm{H}), 3.53-3.36(\mathrm{~m}, 1 \mathrm{H}), 2.72-2.49(\mathrm{~m}$, 2H), 1.92-1.51 (m, 8H), 0.99 (t, J 7.20 Hz, 3H). ${ }^{13} \mathrm{C}$ NMR $\left(\mathrm{CDCl}_{3}, 100 \mathrm{MHz}\right) \delta: 98.80,67.26,62.26,51.81,35.98$, 29.21, 25.44, 19.59. HRMS calc. for $\mathrm{C}_{8} \mathrm{H}_{16} \mathrm{OS}: 160.0921$. Found: 160.0918 .

\section{2-(Benzylthio)-tetrahydro-2H-pyran (5b)}

Yield: $0.191 \mathrm{~g}(91 \%) .{ }^{1} \mathrm{H} \mathrm{NMR}\left(\mathrm{CDCl}_{3}, 400 \mathrm{MHz}\right)$ $\delta: 7.33-7.20(\mathrm{~m}, 5 \mathrm{H}), 4.72-4.69(\mathrm{~m}, 1 \mathrm{H}), 4.14-4.08(\mathrm{~m}, 1 \mathrm{H})$, 3.87-3.81 (m, 1H), 3.75-3.70 (m, 1H), 3.53-3.47 (m, 1H), 1.89-1.75 (m, 2H), 1.67-1.49 (m, 4H). ${ }^{13} \mathrm{C}$ NMR $\left(\mathrm{CDCl}_{3}\right.$, $100 \mathrm{MHz}) \delta: 128.26,128.65,128.09,126.49,80.14$, 63.80, 34.38, 33.59, 30.49, 25.37, 21.27. HRMS calc. for $\mathrm{C}_{12} \mathrm{H}_{16}$ OS: 208.0921. Found: 208.0924.

\section{2-(4-Methoxyphenylthio)-tetrahydro-2H-pyran (5c)}

Yield: $0.194 \mathrm{~g}(87 \%) .{ }^{1} \mathrm{H}$ NMR $\left(\mathrm{CDCl}_{3}, 400 \mathrm{MHz}\right)$ $\delta: 7.43(\mathrm{~d}, J 8.8 \mathrm{~Hz}, 2 \mathrm{H}), 6.84(\mathrm{~d}, J 8.8 \mathrm{~Hz}, 2 \mathrm{H}), 5.04-4.98$ $(\mathrm{m}, 1 \mathrm{H}), 4.23-4.12(\mathrm{~m}, 1 \mathrm{H}), 3.79(\mathrm{~s}, 3 \mathrm{H}), 3.59-3.48(\mathrm{~m}, 1 \mathrm{H})$, 2.04-1.77 (m, 2H), 1.72-1.57 (m, 4H). ${ }^{13} \mathrm{C}$ NMR $\left(\mathrm{CDCl}_{3}\right.$, $100 \mathrm{MHz}) \delta: 158.96,133.94,124.85,114.00,85.82,64.22$, 54.81, 31.15, 25.21, 21.37. HRMS calc. for $\mathrm{C}_{12} \mathrm{H}_{16} \mathrm{O}_{2} \mathrm{~S}$ : 224.0871. Found: 224.0874.

\section{2-(2-Furan-2-ylmethylthio)-tetrahydro-2H-pyran (5d)}

Yield: $0.120 \mathrm{~g}(61 \%) .{ }^{1} \mathrm{H}$ NMR $\left(\mathrm{CDCl}_{3}, 400 \mathrm{MHz}\right)$ $\delta: 7.35(\mathrm{~s}, 1 \mathrm{H}), 6.30-6.29(\mathrm{~m}, 1 \mathrm{H}), 6.17(\mathrm{~d}, J 2.6 \mathrm{~Hz}, 1 \mathrm{H})$, $4.87-4.85(\mathrm{~m}, 1 \mathrm{H}), 4.12-4.06(\mathrm{~m}, 1 \mathrm{H}), 3.91(\mathrm{~d}, J 14.6 \mathrm{~Hz}$, $1 \mathrm{H}), 3.69(\mathrm{~d}, J 14.6 \mathrm{~Hz}, 1 \mathrm{H}), 3.55-3.49(\mathrm{~m}, 1 \mathrm{H}), 1.94-1.54$ $(\mathrm{m}, 6 \mathrm{H}) .{ }^{13} \mathrm{C} \mathrm{NMR}\left(\mathrm{CDCl}_{3}, 100 \mathrm{MHz}\right) \delta: 151.68,141.97$, $110.27,107.28,80.84,64.02,30.64,25.89,25.56,21.39$. HRMS calc. for $\mathrm{C}_{10} \mathrm{H}_{14} \mathrm{O}_{2} \mathrm{~S}:$ 198.0714. Found: 198.0716.

\section{2-(2-Chlorophenylthio)-tetrahydro-2H-pyran (5e)}

Yield: $0.194 \mathrm{~g}(85 \%) .{ }^{1} \mathrm{H}$ NMR $\left(\mathrm{CDCl}_{3}, 400 \mathrm{MHz}\right)$ $\delta: 7.59(\mathrm{dd}, J 6.0 / 1.7 \mathrm{~Hz}, 1 \mathrm{H}), 7.36(\mathrm{dd}, J 6.1 / 1.4 \mathrm{~Hz}, 1 \mathrm{H})$, 7.26-7.07 (m, 2H), $5.37(\mathrm{t}, J 4.4 \mathrm{~Hz}, 1 \mathrm{H}), 4.22-4.11(\mathrm{~m}, 1 \mathrm{H})$, 3.67-3.56 (m, $1 \mathrm{H}), 2.17-1.59(\mathrm{~m}, 6 \mathrm{H}) .{ }^{13} \mathrm{C} \mathrm{NMR}\left(\mathrm{CDCl}_{3}\right.$, $100 \mathrm{MHz}) \delta: 135.02,133.27,129.90,129.12,126.91$, $126.65,83.21,63.67,31.05,25.19,20.96$. HRMS calc. for $\mathrm{C}_{11} \mathrm{H}_{13}$ ClOS: 228.0375. Found: 228.0371.

\section{2-(4-Chlorobenzylthio)-tetrahydro-2H-pyran (5f)}

Yield: $0.218 \mathrm{~g}(90 \%) .{ }^{1} \mathrm{H} \mathrm{NMR}\left(\mathrm{CDCl}_{3}, 400 \mathrm{MHz}\right)$ $\delta: ~ 7.28-7.15(\mathrm{~m}, 4 \mathrm{H}), 4.69-4.66(\mathrm{~m}, 1 \mathrm{H}), 4.11-4.06(\mathrm{~m}, 1 \mathrm{H})$, 3.83-3.75 (m, 1H), 3.69-3.64 (m, 1H), 3.51-3.46 (m, 1H), 1.89$1.46(\mathrm{~m}, 6 \mathrm{H}) .{ }^{13} \mathrm{C} \mathrm{NMR}\left(\mathrm{CDCl}_{3}, 100 \mathrm{MHz}\right) \delta: 136.93,132.31$, $130.08,128.28,80.18,63.88,32.98,30.50,25.40,21.29$. HRMS calc. for $\mathrm{C}_{12} \mathrm{H}_{15}$ ClOS: 242.0532. Found: 242.0535 . 


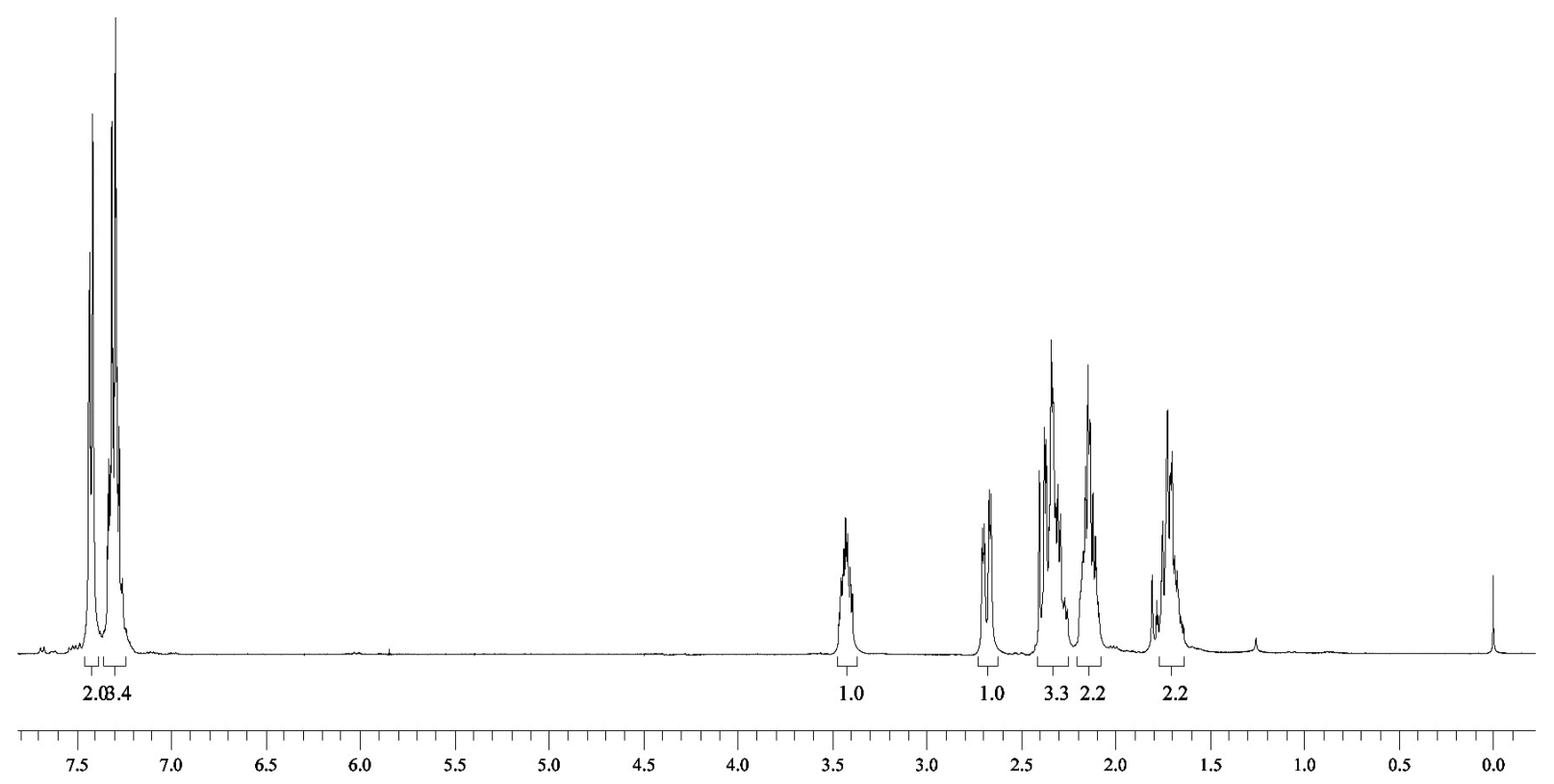

Figure S1. ${ }^{1} \mathrm{H}$ NMR spectra of compound $3 \mathbf{a}$ in $\mathrm{CDCl}_{3}$ at $200 \mathrm{MHz}$.

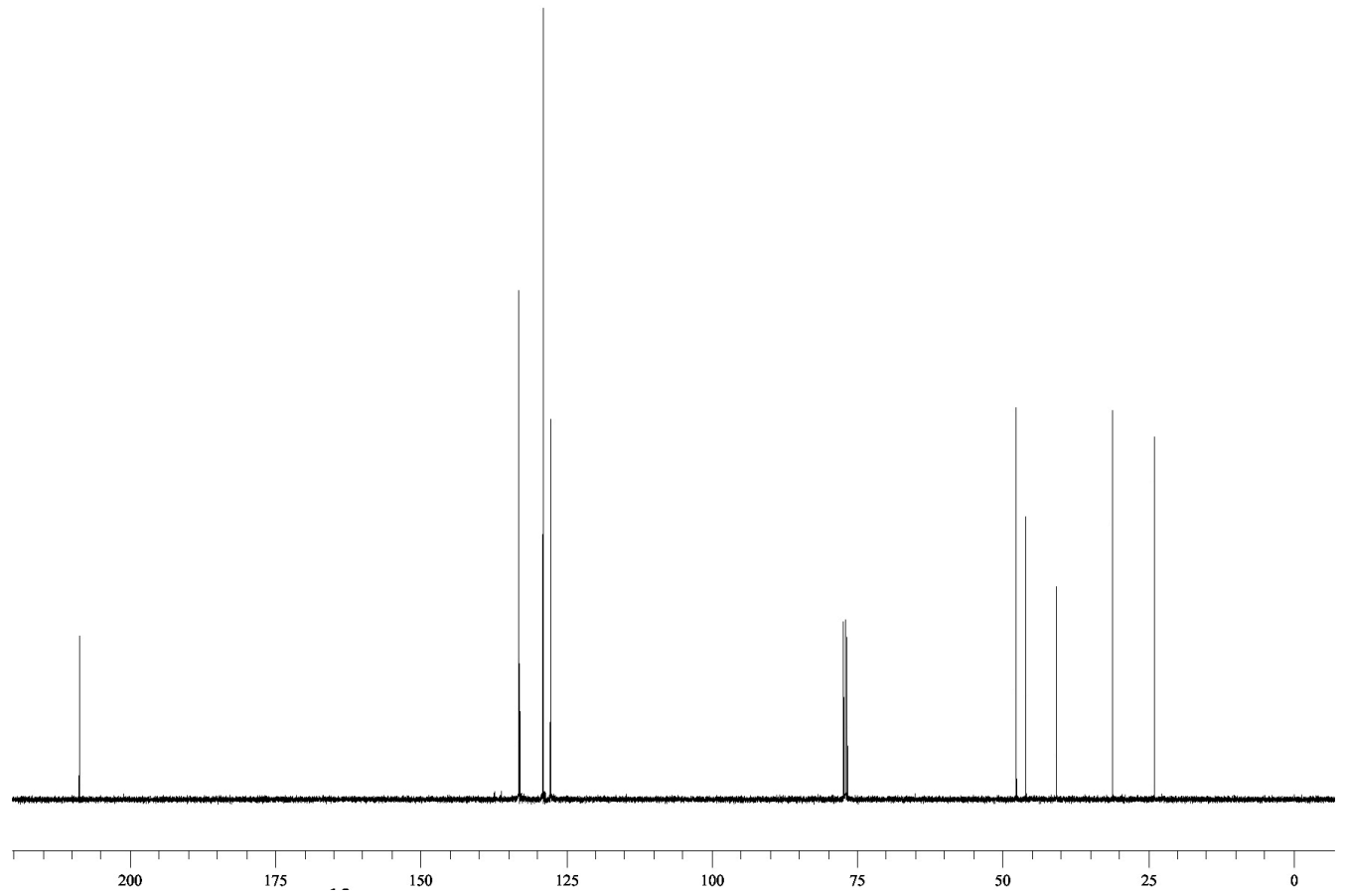

Figure S2. ${ }^{13} \mathrm{C}$ NMR spectra of compound 3a in $\mathrm{CDCl}_{3}$ at $100 \mathrm{MHz}$. 


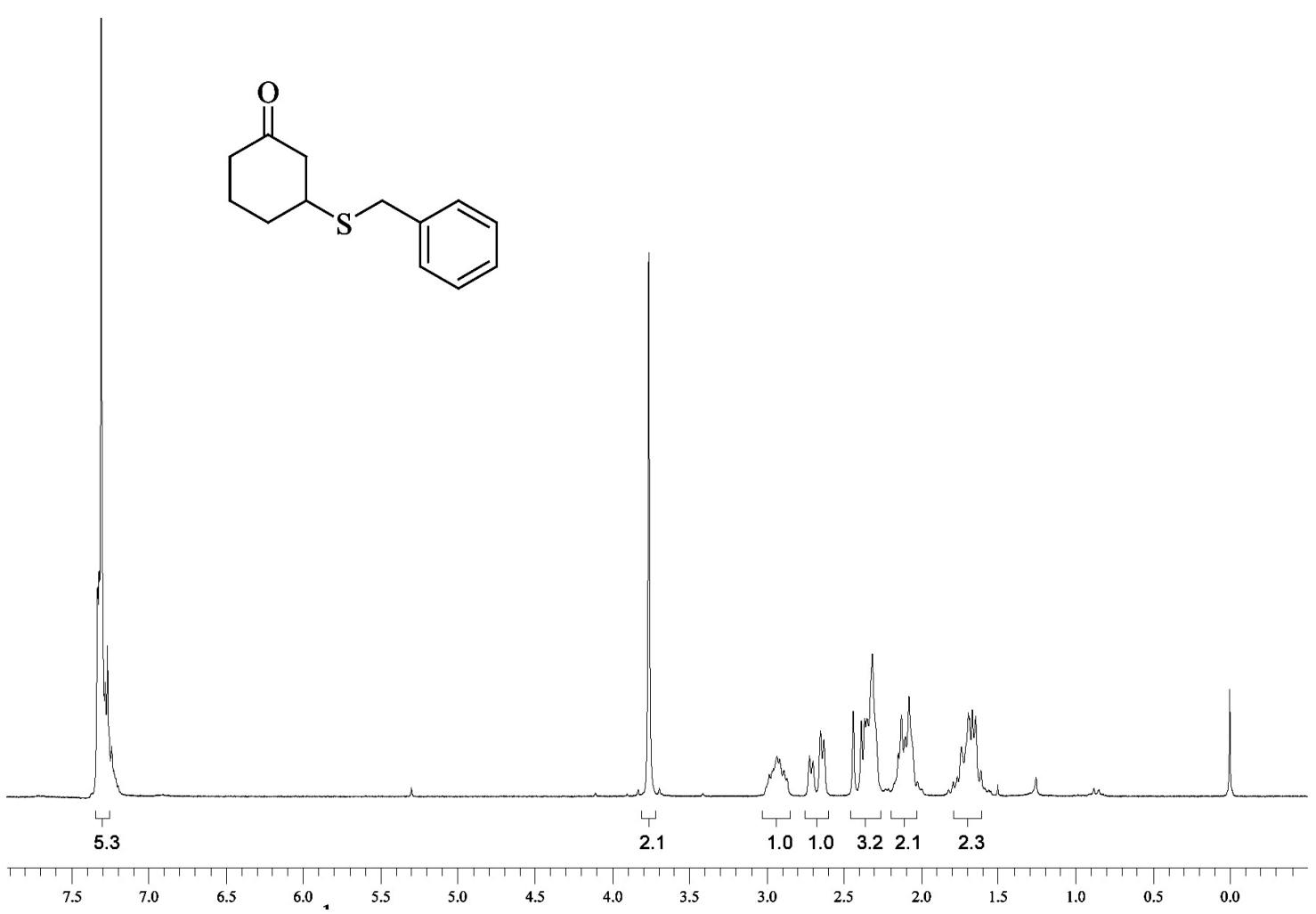

Figure S3. ${ }^{1} \mathrm{H}$ NMR spectra of compound $\mathbf{3 c}$ in $\mathrm{CDCl}_{3}$ at $200 \mathrm{MHz}$.

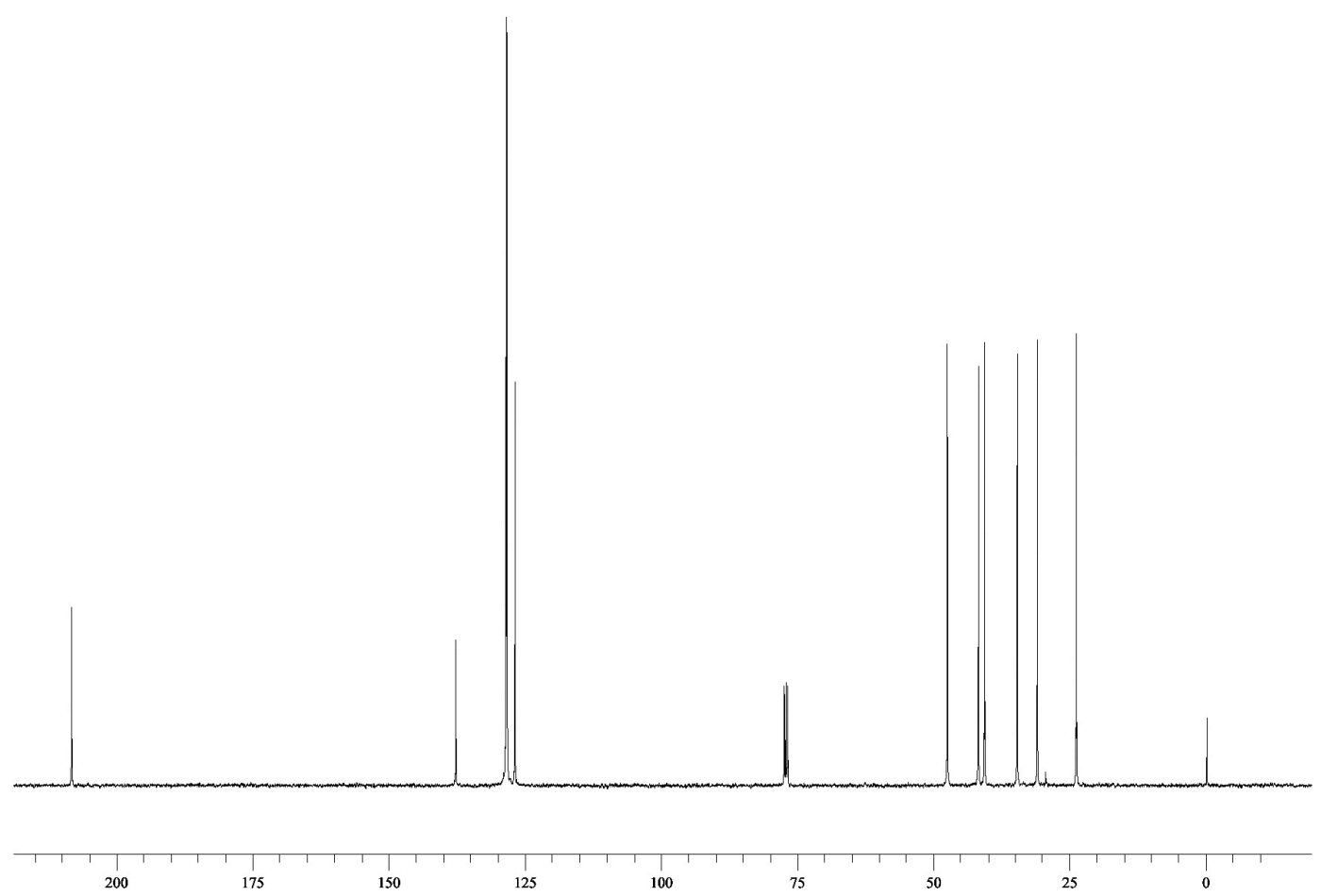

Figure S4. ${ }^{13} \mathrm{C}$ NMR spectra of compound 3a in $\mathrm{CDCl}_{3}$ at $100 \mathrm{MHz}$. 
<smiles>CC(C)(C)SC1CCCC(=O)C1</smiles>
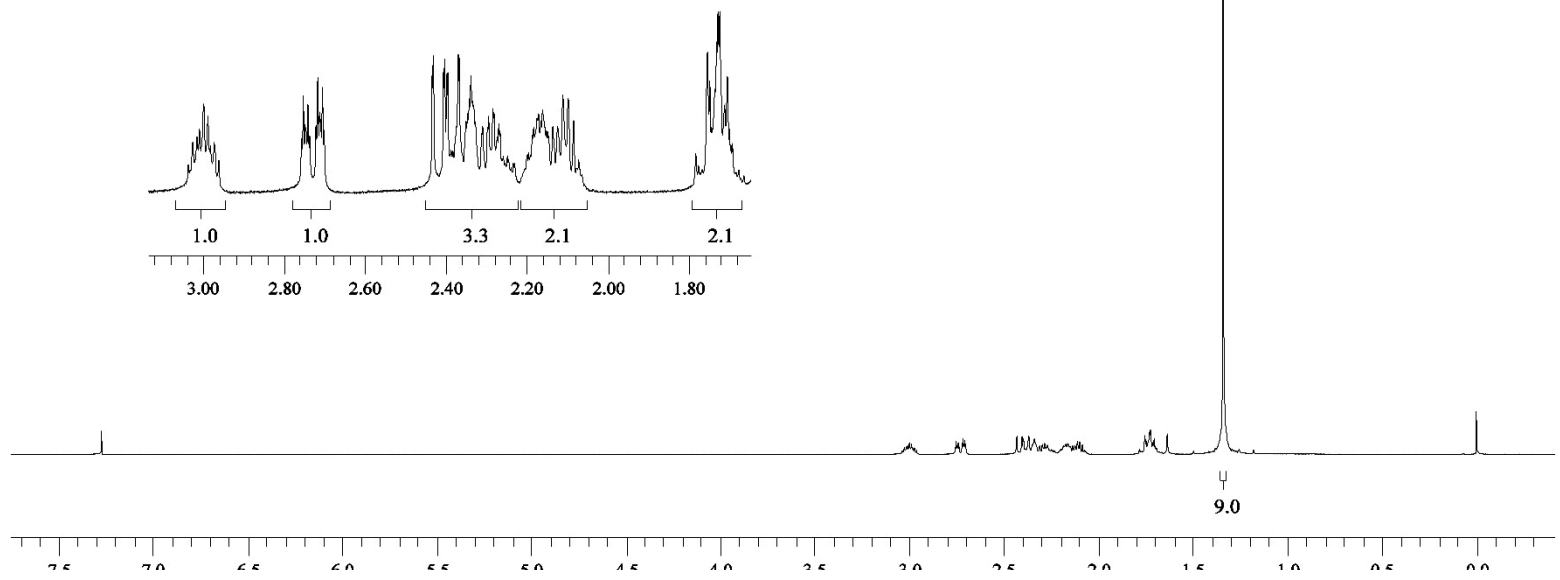

Figure S5. ${ }^{~} \mathrm{H}$ NMR spectra of compound $\mathbf{3 d}$ in $\mathrm{CDCl}_{3}$ at $200 \mathrm{MHz}$.

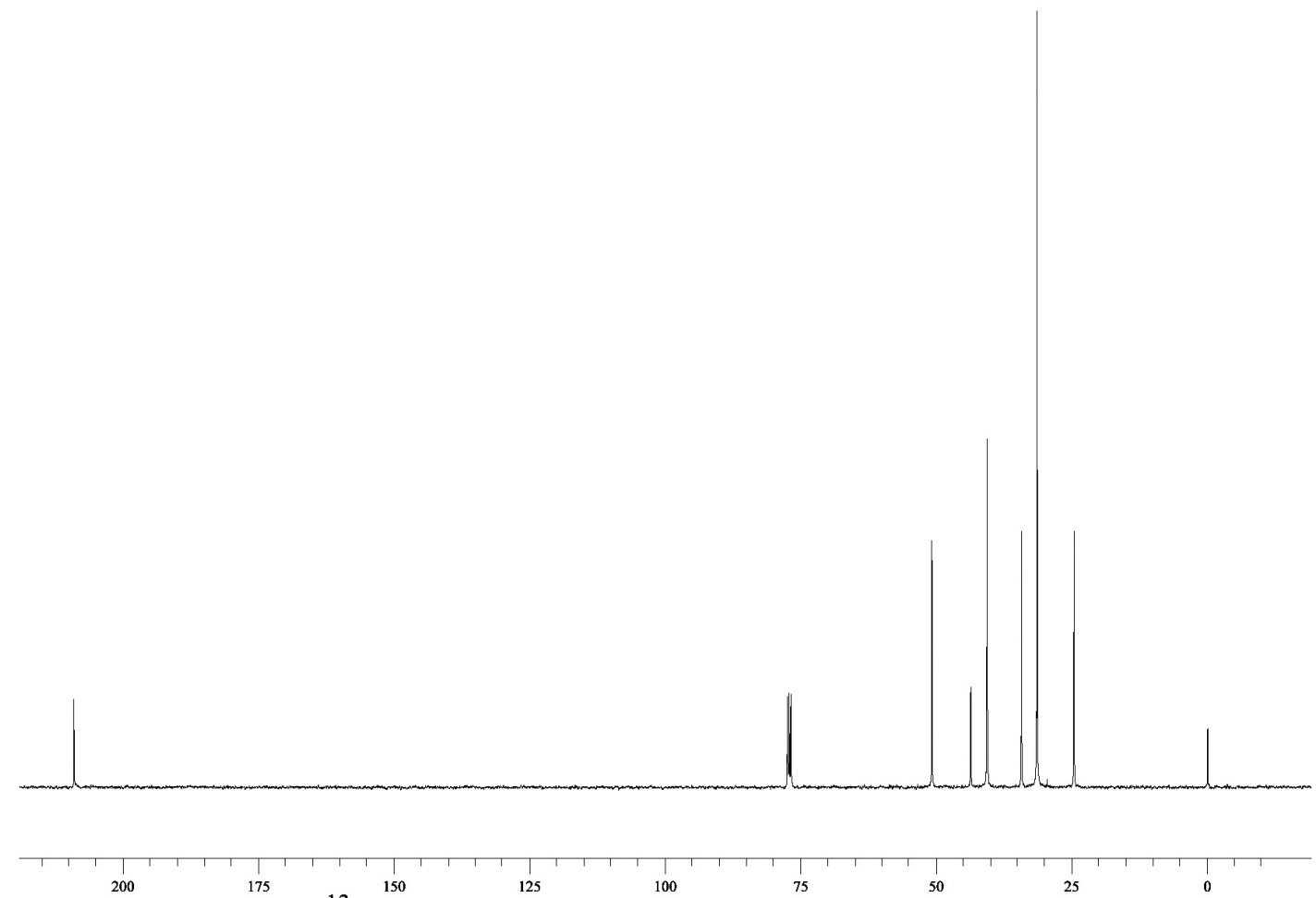

Figure S6. ${ }^{13} \mathrm{C}$ NMR spectra of compound $\mathbf{3 d}$ in $\mathrm{CDCl}_{3}$ at $100 \mathrm{MHz}$. 


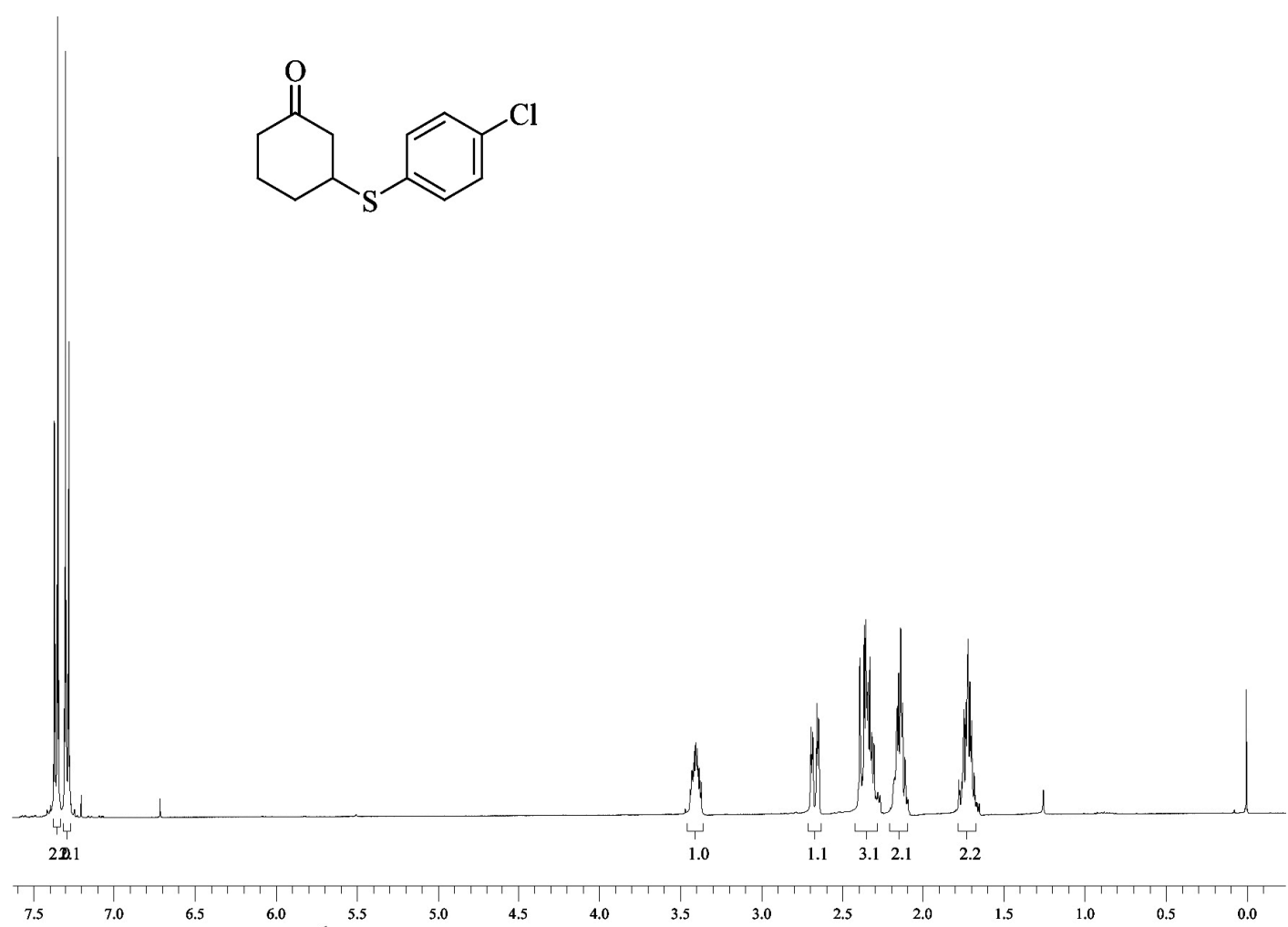

Figure S7. ${ }^{1} \mathrm{H}$ NMR spectra of compound $\mathbf{3 h}$ in $\mathrm{CDCl}_{3}$ at $400 \mathrm{MHz}$.

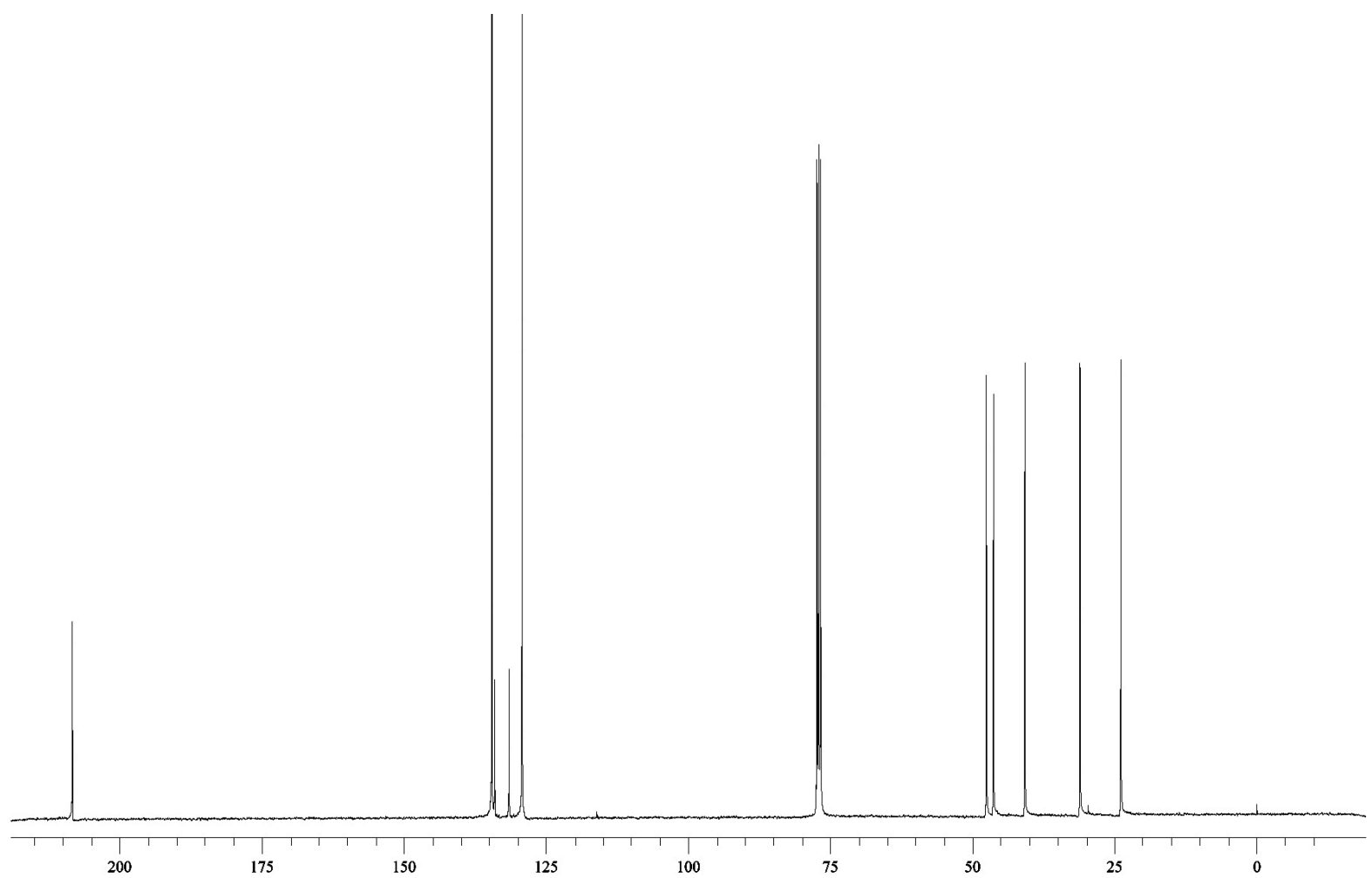

Figure S8. ${ }^{13} \mathrm{C}$ NMR spectra of compound $\mathbf{3 h}$ in $\mathrm{CDCl}_{3}$ at $100 \mathrm{MHz}$. 


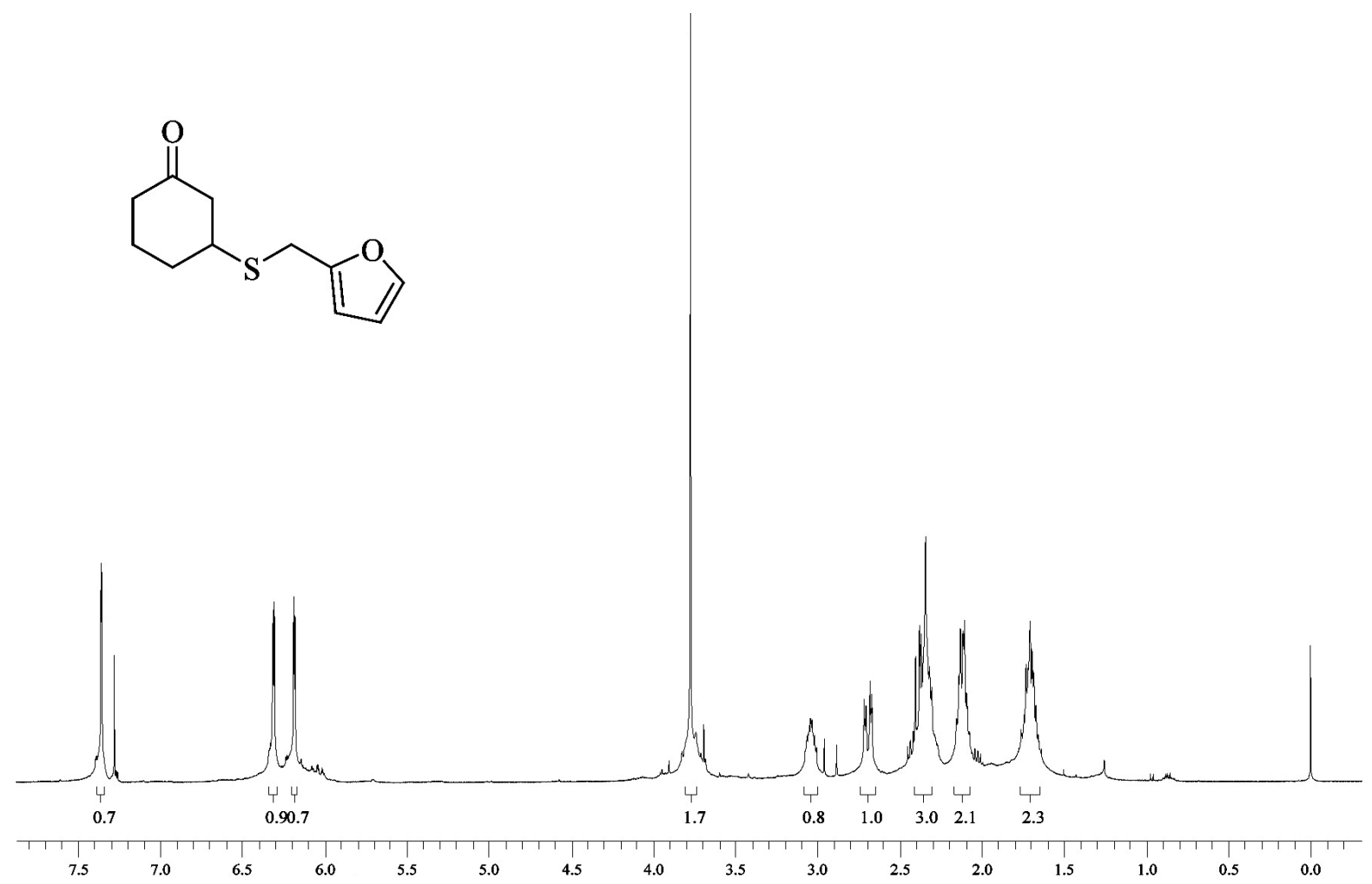

Figure S9. ${ }^{1} \mathrm{H}$ NMR spectra of compound $3 \mathbf{i}$ in $\mathrm{CDCl}_{3}$ at $200 \mathrm{MHz}$.

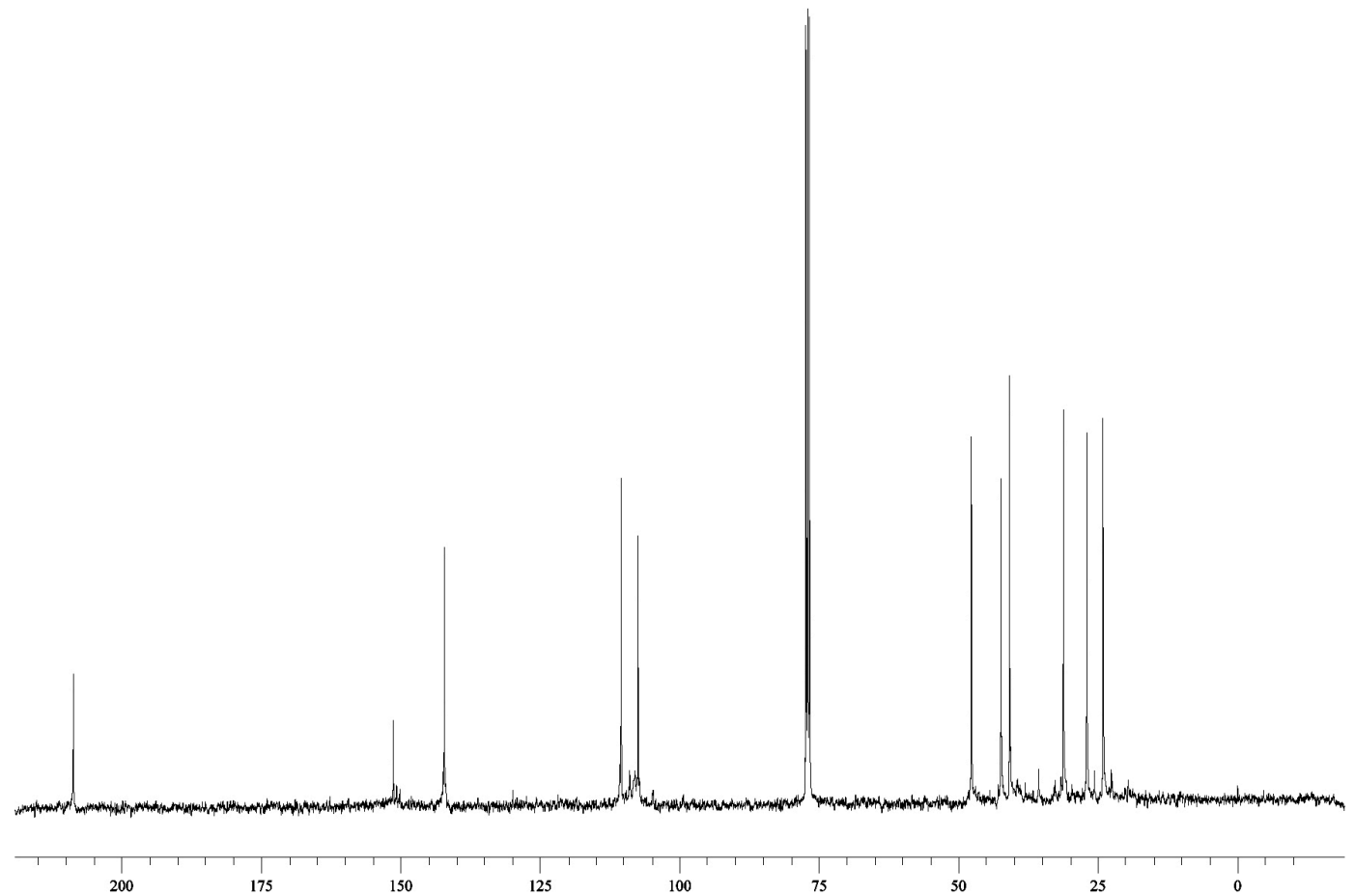

Figure S10. ${ }^{13} \mathrm{C}$ NMR spectra of compound $3 \mathbf{i}$ in $\mathrm{CDCl}_{3}$ at $100 \mathrm{MHz}$. 
10

PhSeBr-Catalyzed Selective Addition of Thiols to $\alpha, \beta$-Unsaturated Carbonyl Compounds

J. Braz. Chem. Soc.

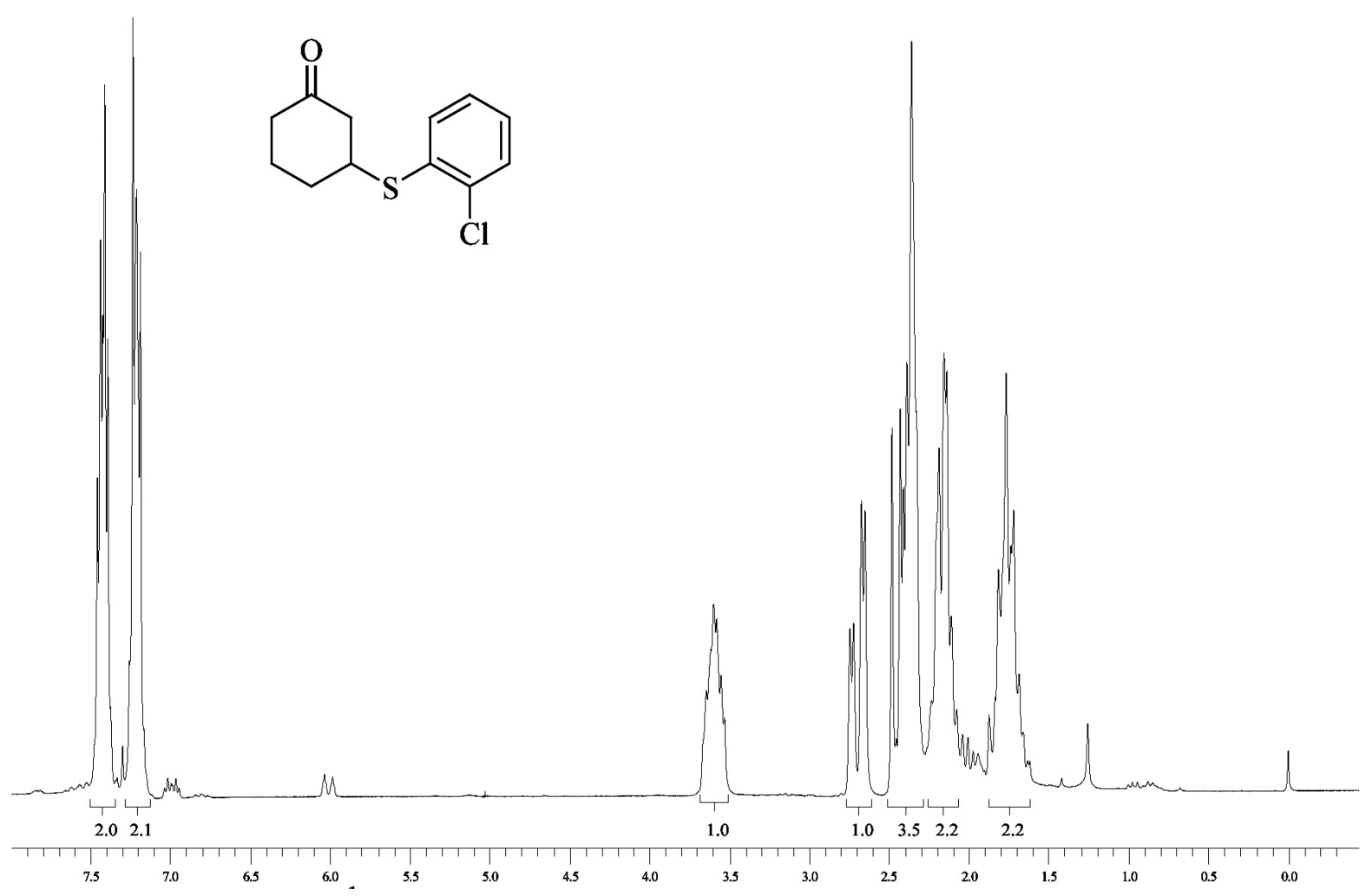

Figure S11. ${ }^{1} \mathrm{H}$ NMR spectra of compound $\mathbf{3 k}$ in $\mathrm{CDCl}_{3}$ at $200 \mathrm{MHz}$.

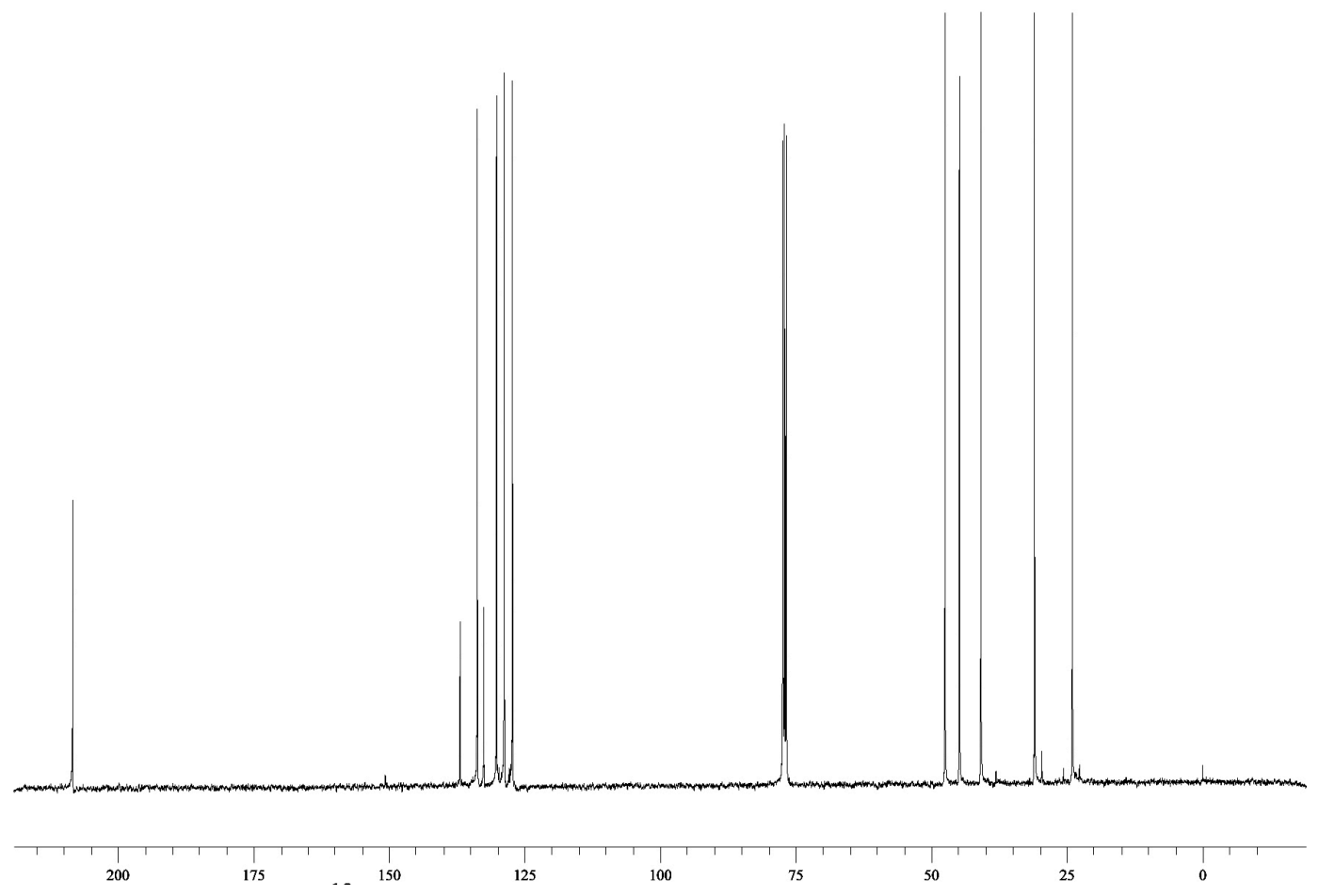

Figure S12. ${ }^{13} \mathrm{C}$ NMR spectra of compound $\mathbf{3 k}$ in $\mathrm{CDCl}_{3}$ at $100 \mathrm{MHz}$. 
Vol. 00, No. 00, 2010

Schneider et al.

11

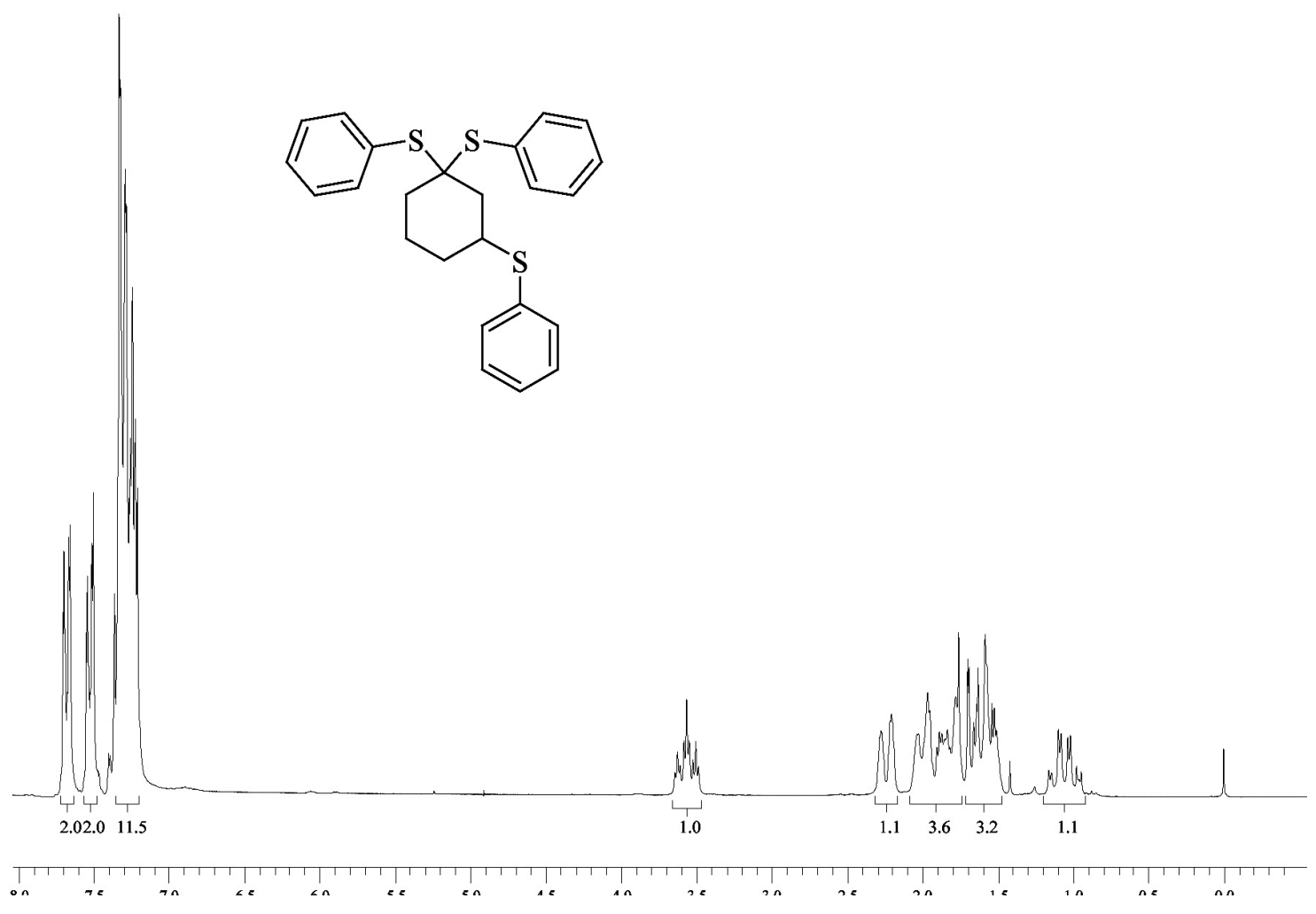

Figure S13. ${ }^{1} \mathrm{H}$ NMR spectra of compound $4 a$ in $\mathrm{CDCl}_{3}$ at $200 \mathrm{MHz}$.
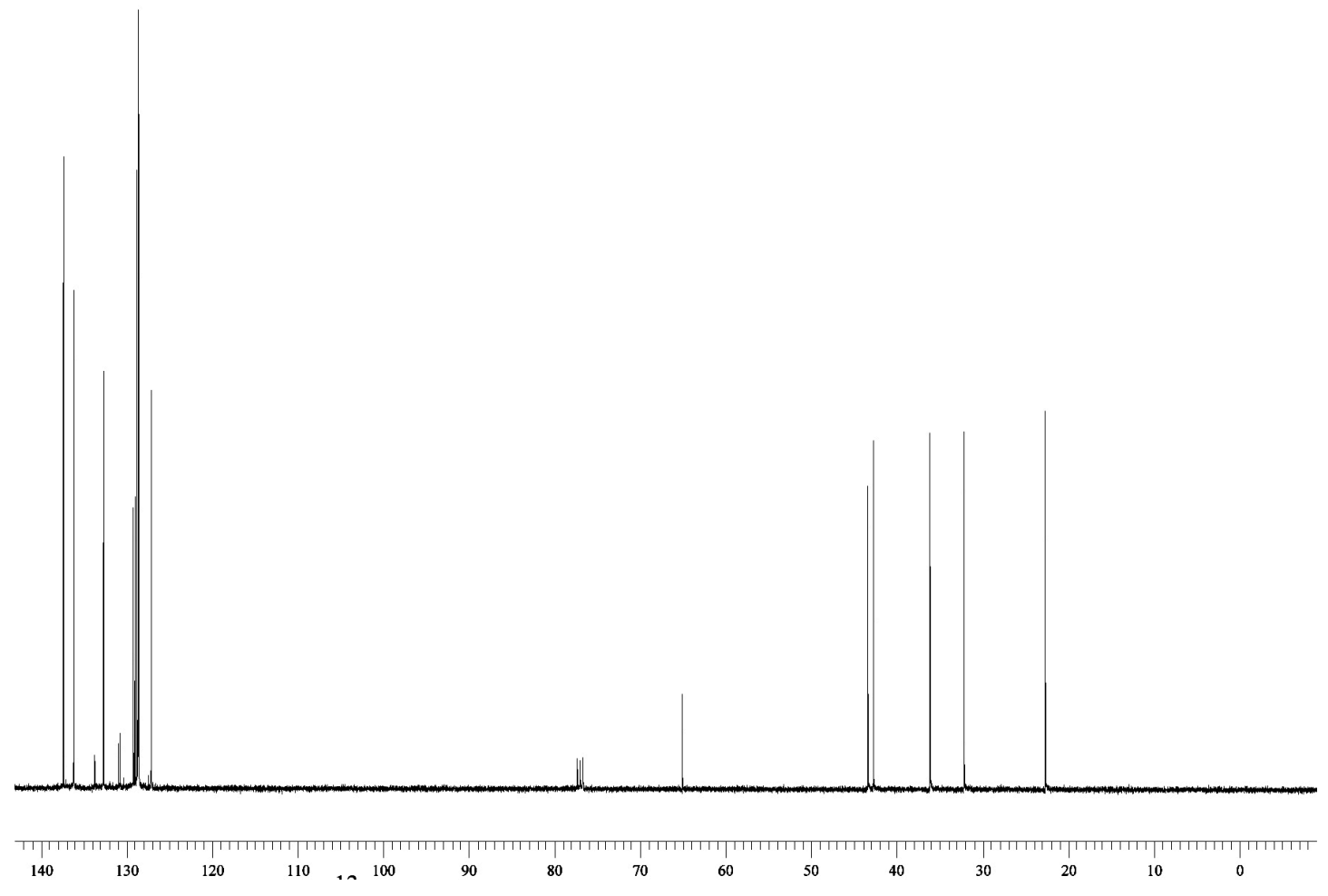

Figure S14. ${ }^{13} \mathrm{C}$ NMR spectra of compound $4 \mathrm{a}$ in $\mathrm{CDCl}_{3}$ at $100 \mathrm{MHz}$. 


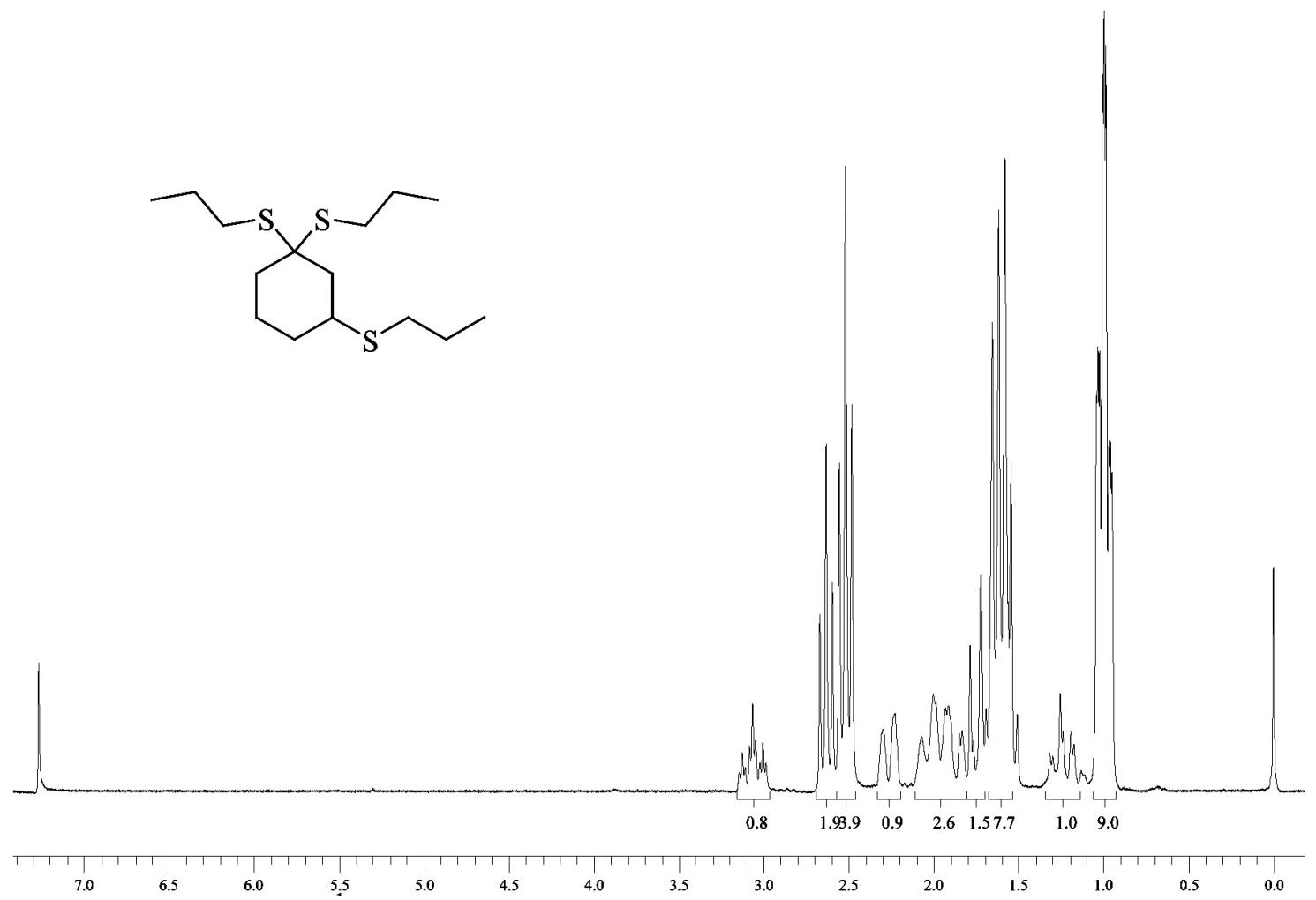

Figure S15. ${ }^{1} \mathrm{H}$ NMR spectra of compound $\mathbf{4 b}$ in $\mathrm{CDCl}_{3}$ at $200 \mathrm{MHz}$.

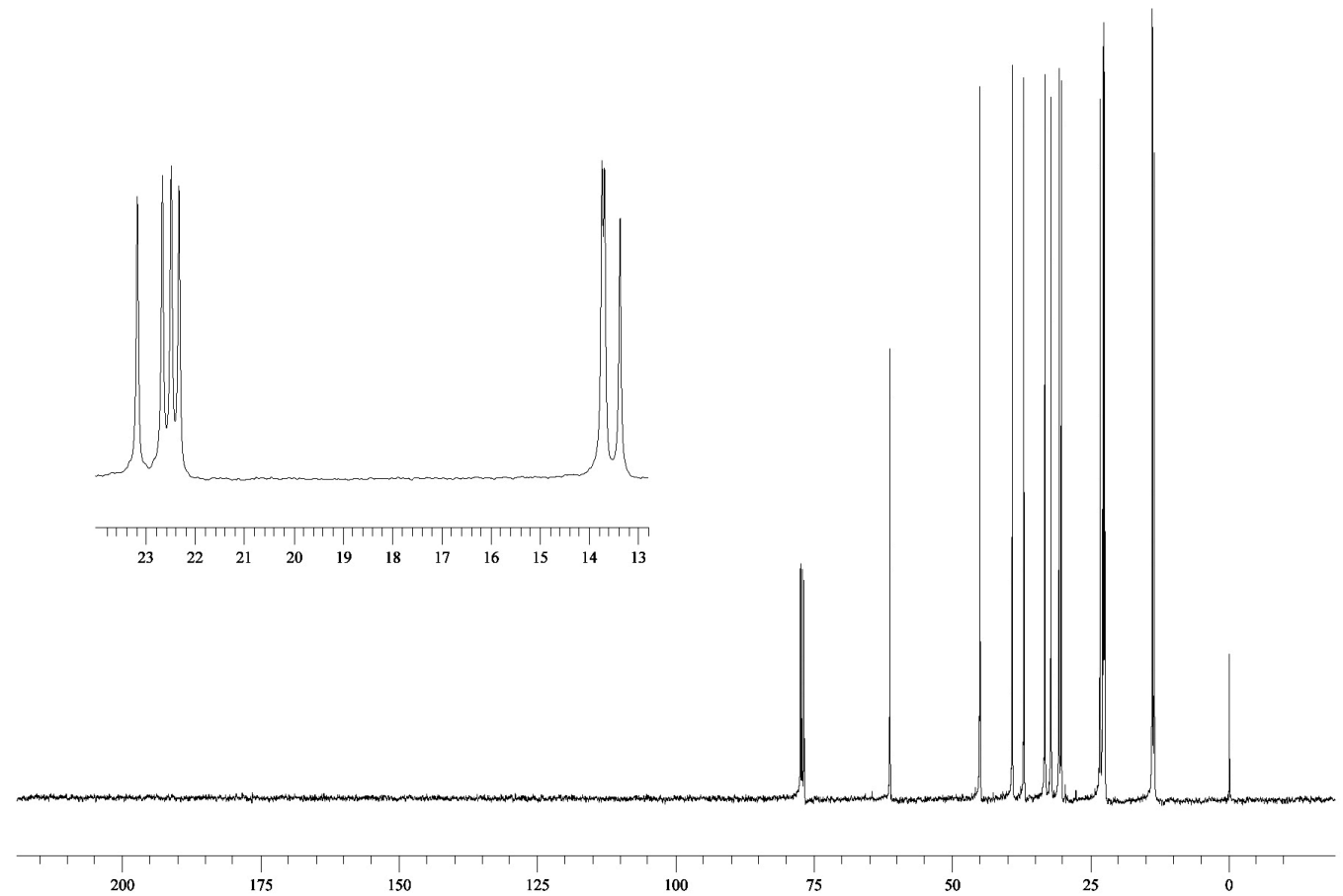

Figure S16. ${ }^{13} \mathrm{C}$ NMR spectra of compound $\mathbf{4 b}$ in $\mathrm{CDCl}_{3}$ at $100 \mathrm{MHz}$. 


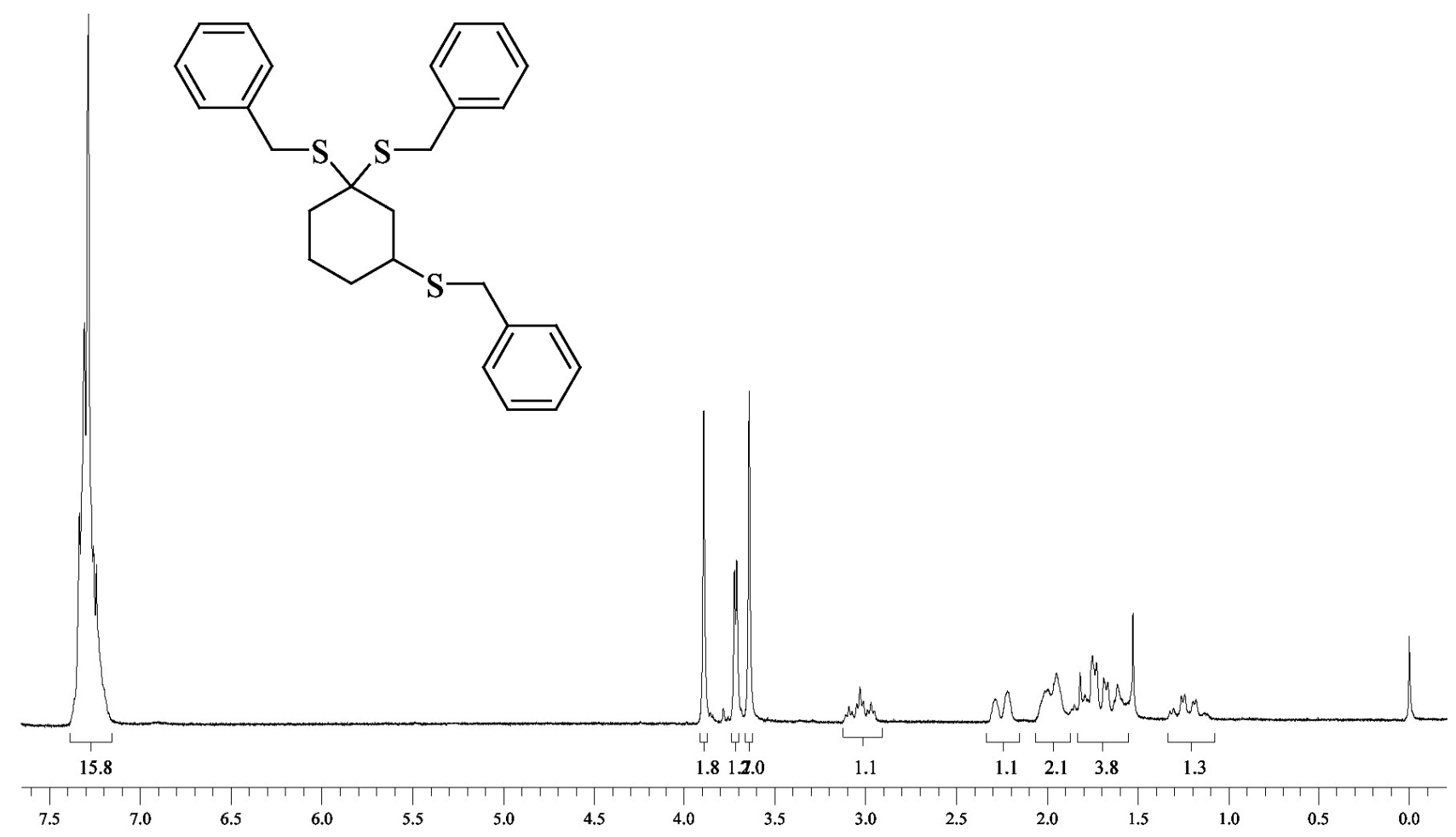

Figure S17. ${ }^{1} \mathrm{H}$ NMR spectra of compound $\mathbf{4 c}$ in $\mathrm{CDCl}_{3}$ at $200 \mathrm{MHz}$.

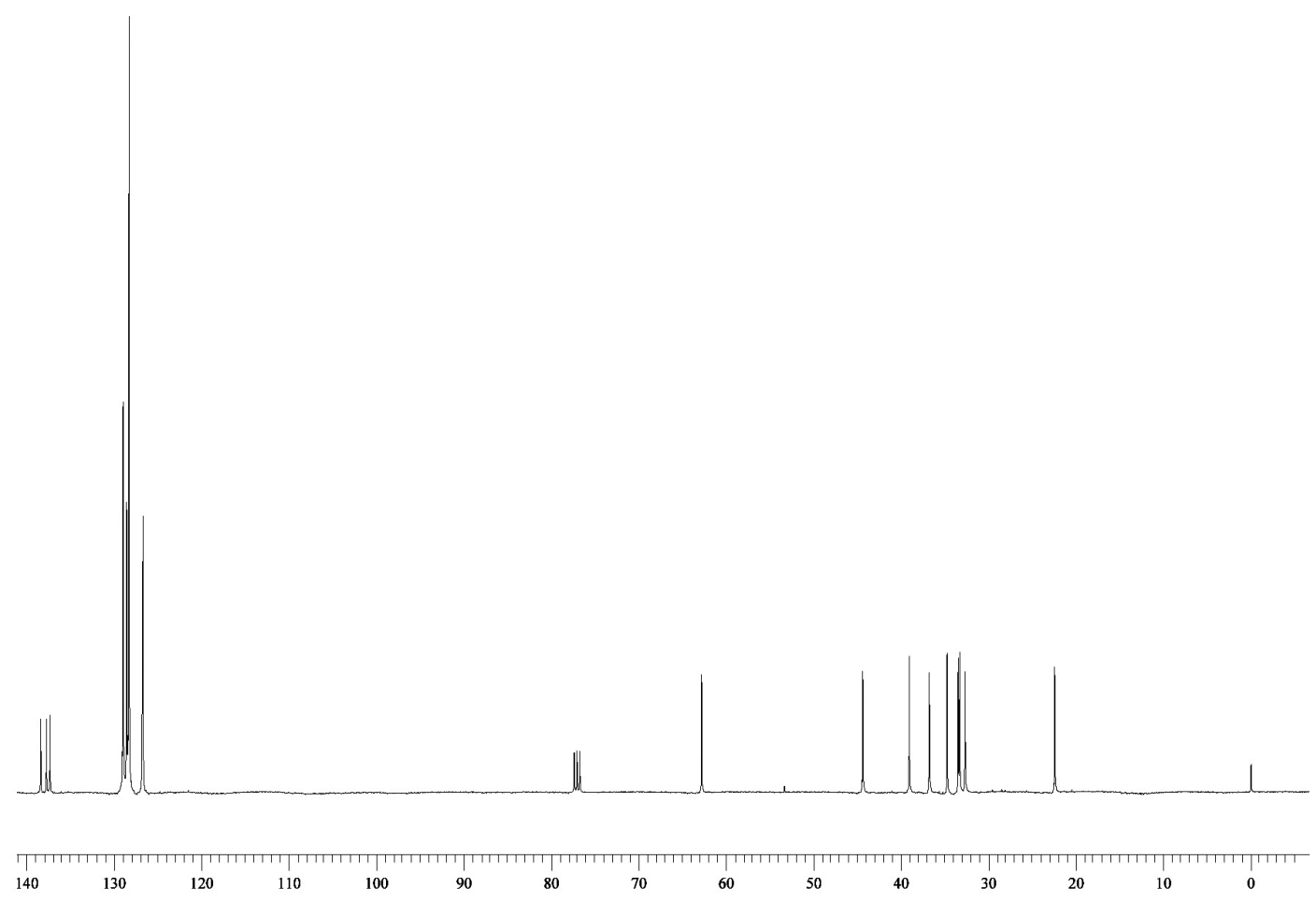

Figure S18. ${ }^{13} \mathrm{C}$ NMR spectra of compound $4 \mathbf{c}$ in $\mathrm{CDCl}_{3}$ at $100 \mathrm{MHz}$. 
<smiles>COc1ccc(SC2CCCC(Sc3ccc(OC)cc3)C2)cc1</smiles>

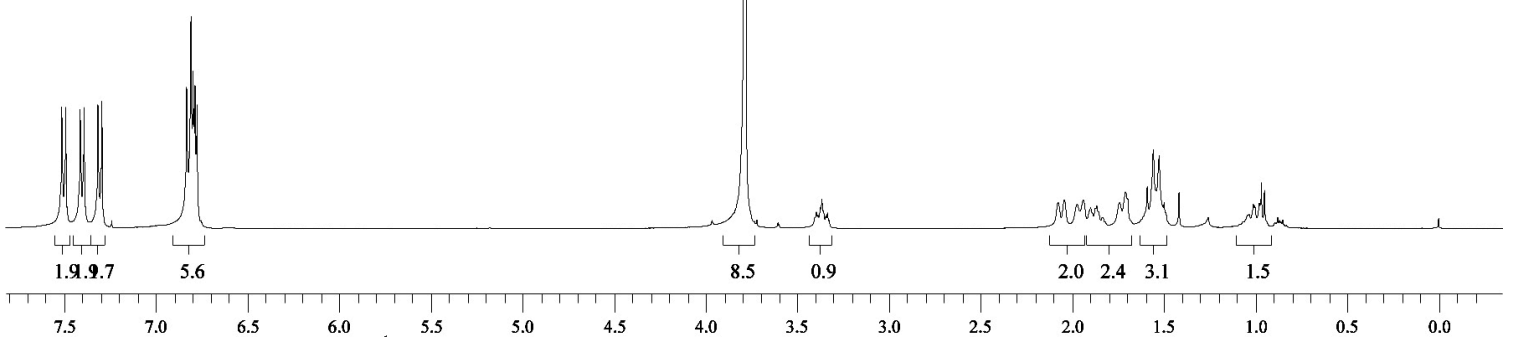

Figure S19. ${ }^{1} \mathrm{H}$ NMR spectra of compound $\mathbf{4 g}$ in $\mathrm{CDCl}_{3}$ at $400 \mathrm{MHz}$.

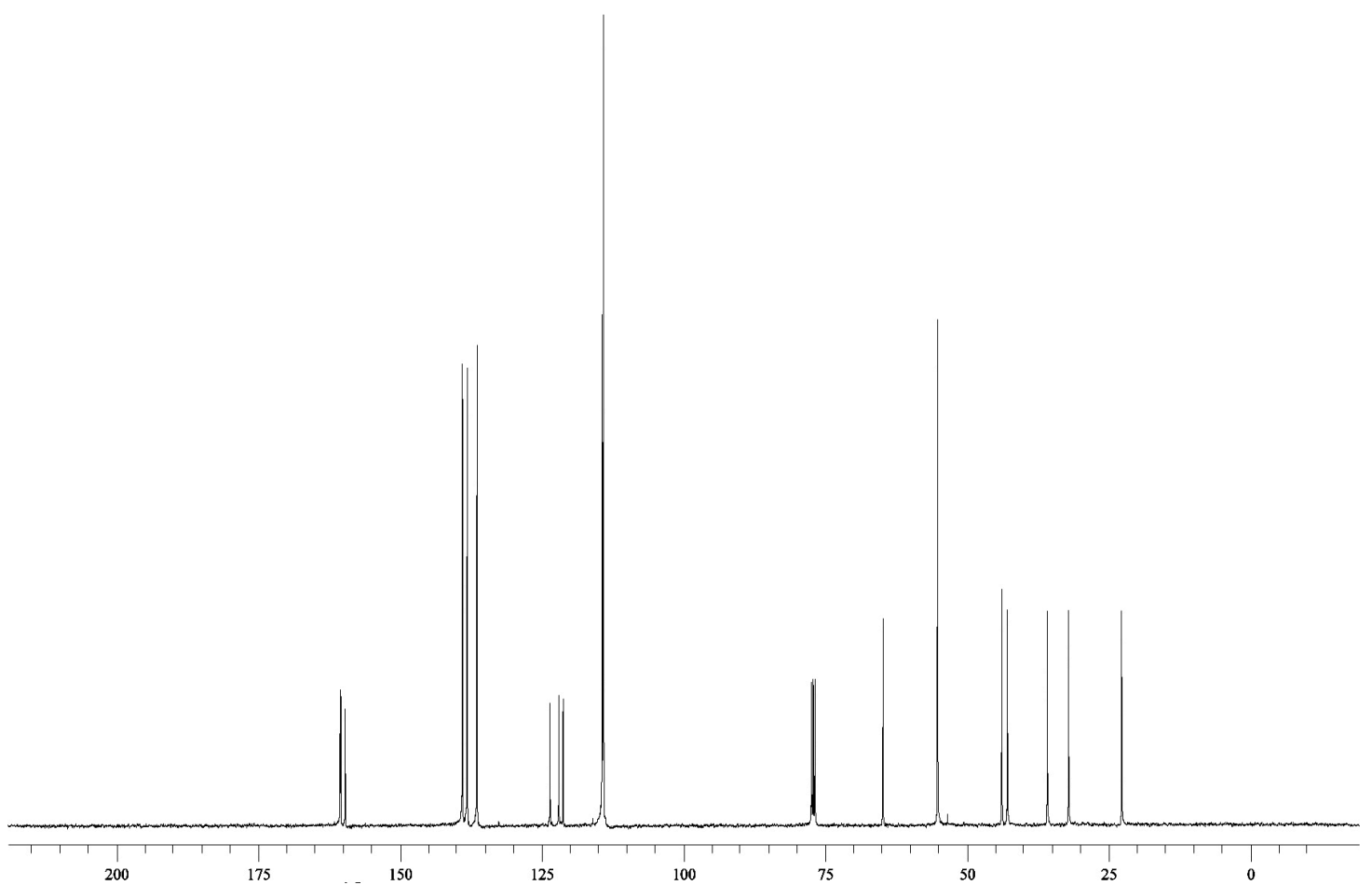

Figure S20. ${ }^{13} \mathrm{C}$ NMR spectra of compound $\mathbf{4 g}$ in $\mathrm{CDCl}_{3}$ at $100 \mathrm{MHz}$. 
Vol. 00, No. 00, 2010

Schneider et al.

15

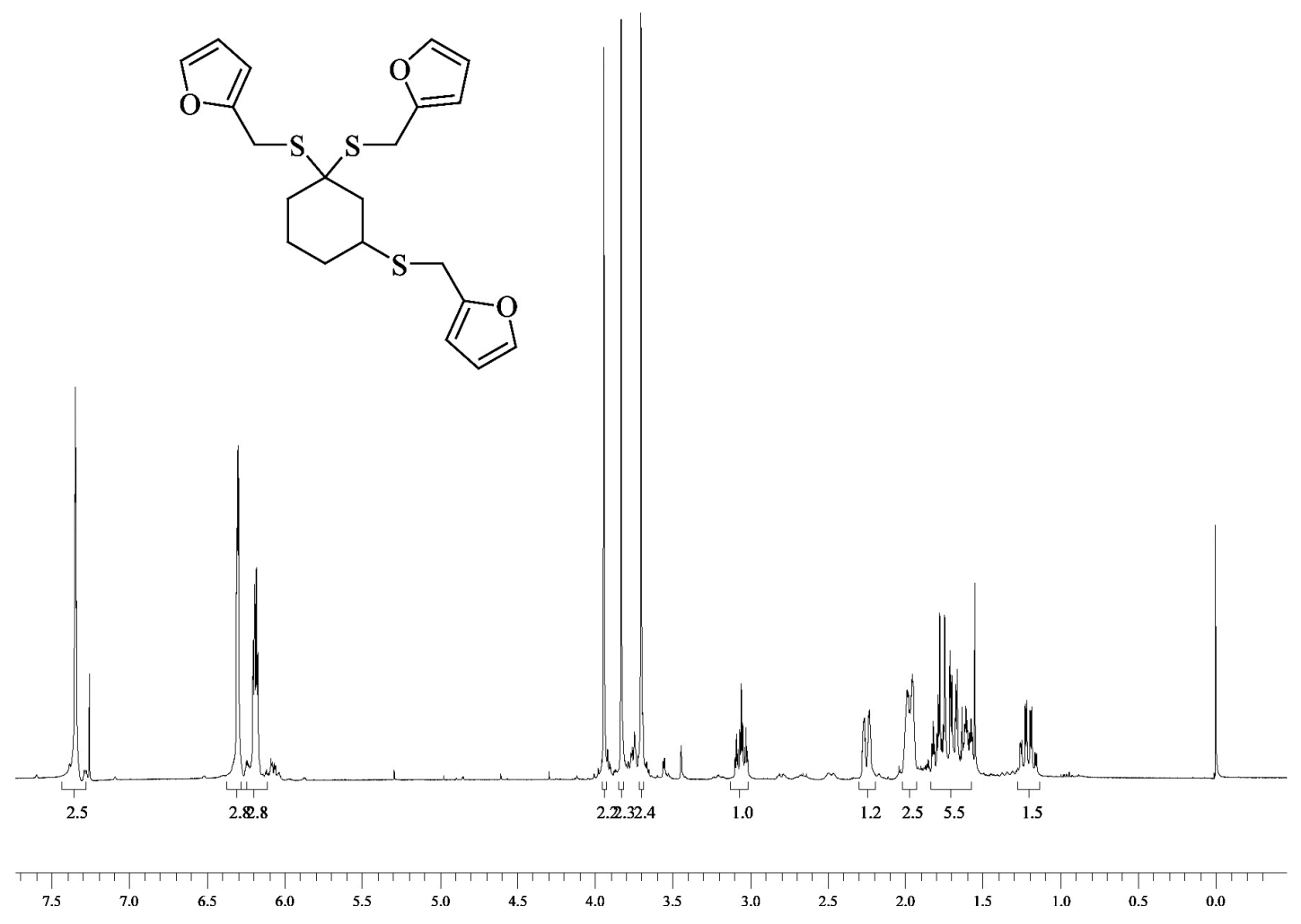

Figure S21. ${ }^{~} \mathrm{H}$ NMR spectra of compound $\mathbf{4 h}$ in $\mathrm{CDCl}_{3}$ at $400 \mathrm{MHz}$.

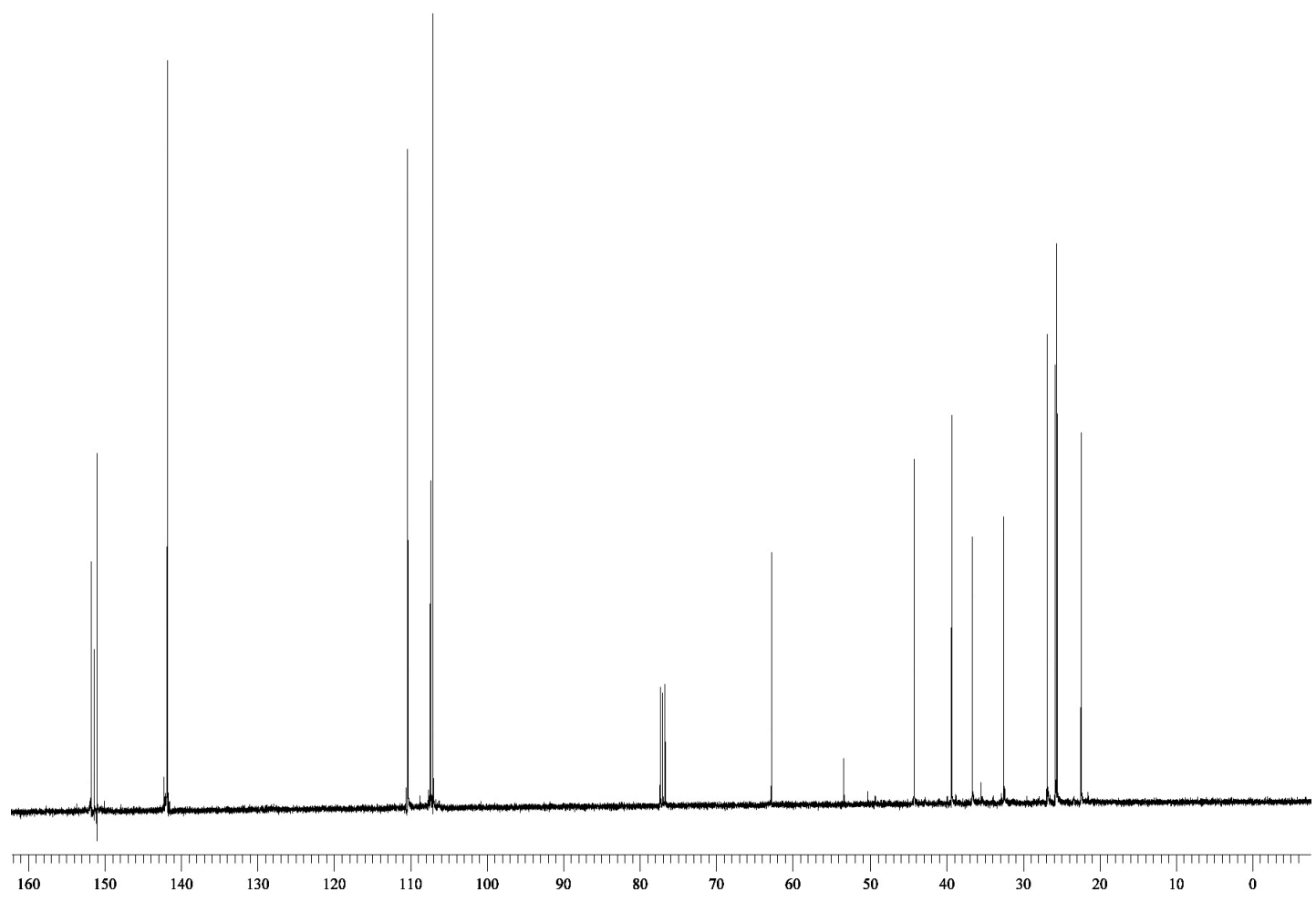

Figure S22. ${ }^{13} \mathrm{C}$ NMR spectra of compound $\mathbf{4 h}$ in $\mathrm{CDCl}_{3}$ at $100 \mathrm{MHz}$. 


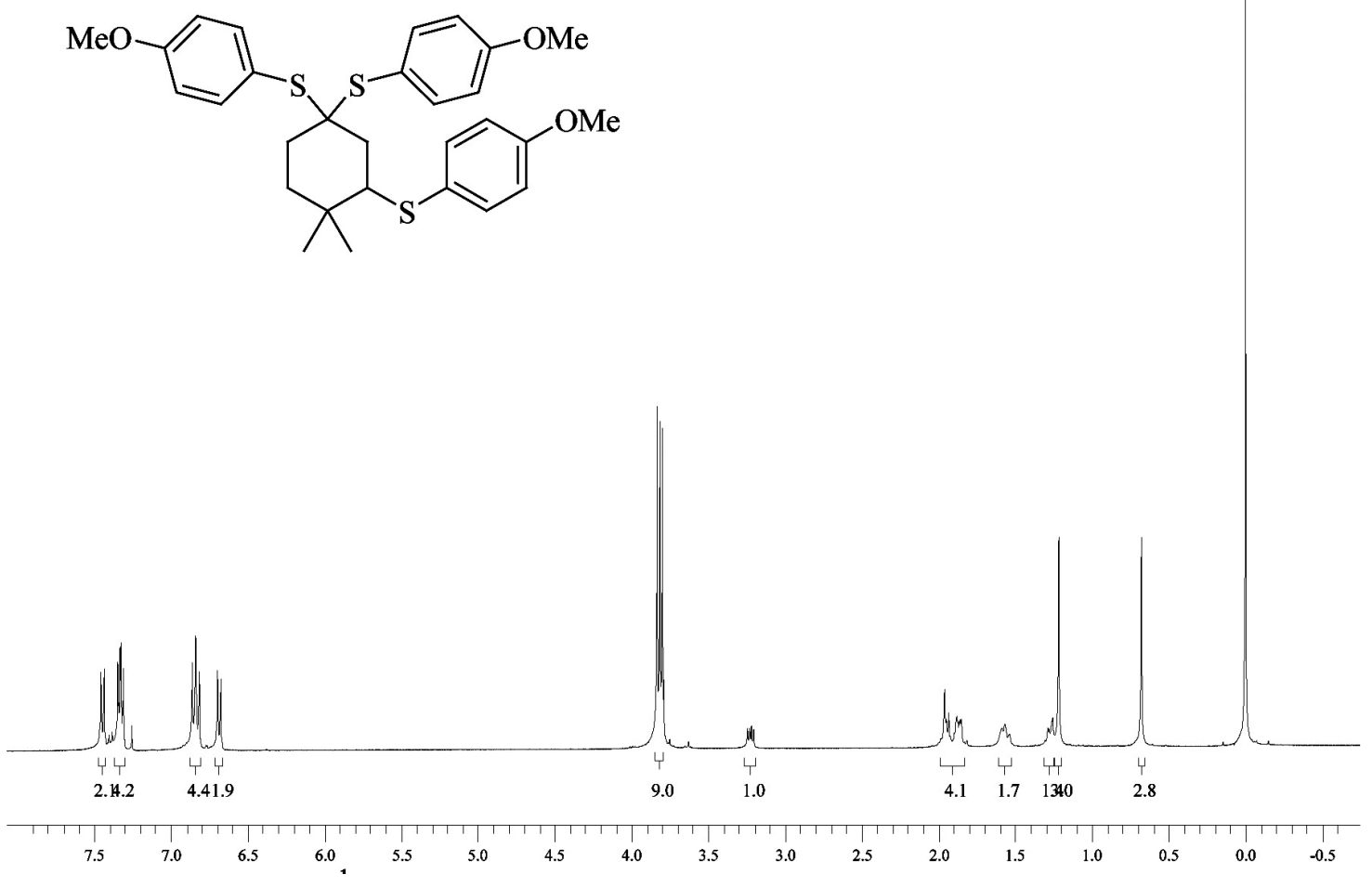

Figure S23. ${ }^{1} \mathrm{H}$ NMR spectra of compound $\mathbf{4 m}$ in $\mathrm{CDCl}_{3}$ at $400 \mathrm{MHz}$.
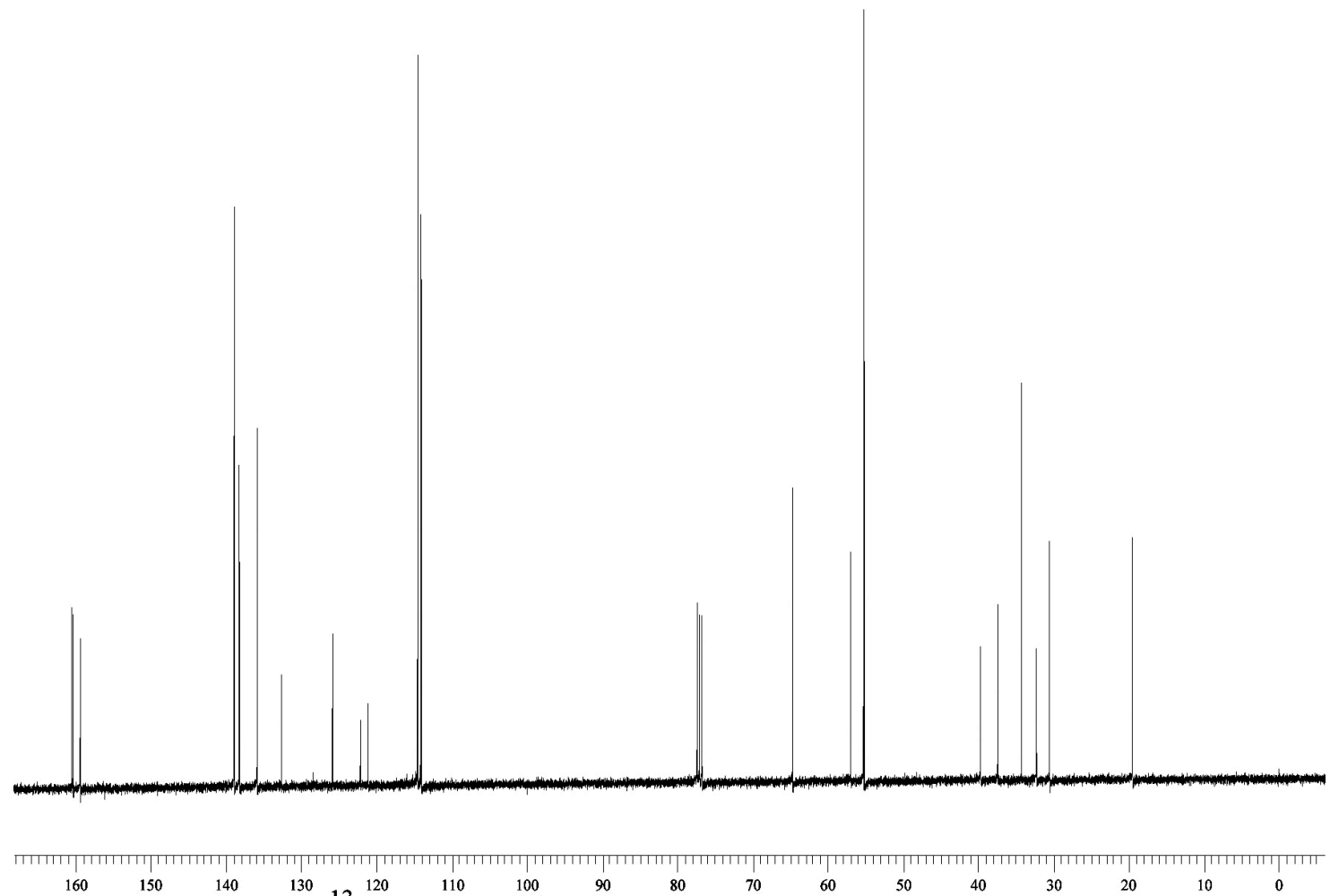

Figure S24. ${ }^{13} \mathrm{C}$ NMR spectra of compound $\mathbf{4 m}$ in $\mathrm{CDCl}_{3}$ at $100 \mathrm{MHz}$. 
<smiles>COc1ccc(SC2CCCCO2)cc1</smiles>

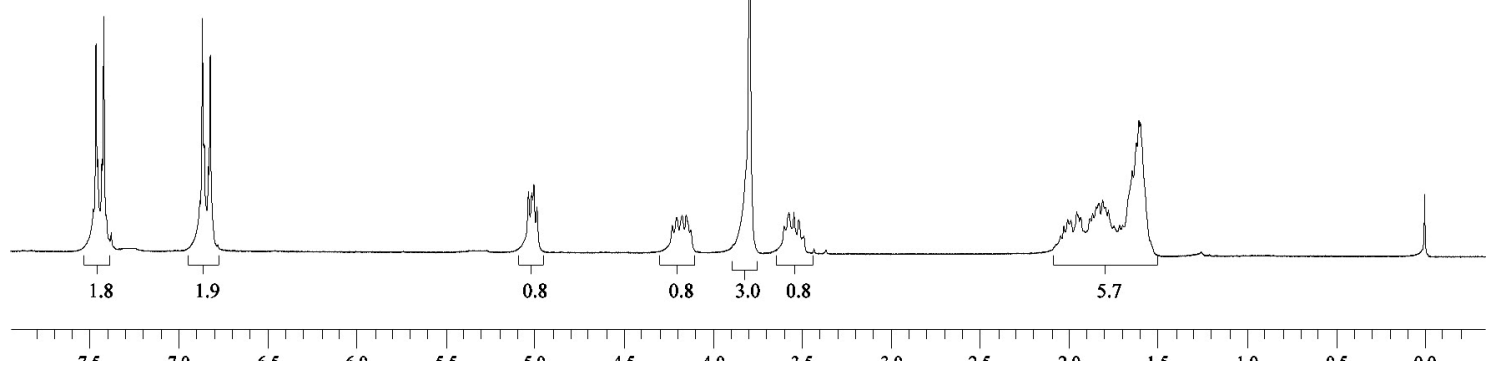

Figure S25. ${ }^{1} \mathrm{H}$ NMR spectra of compound $\mathbf{5 c}$ in $\mathrm{CDCl}_{3}$ at $400 \mathrm{MHz}$.

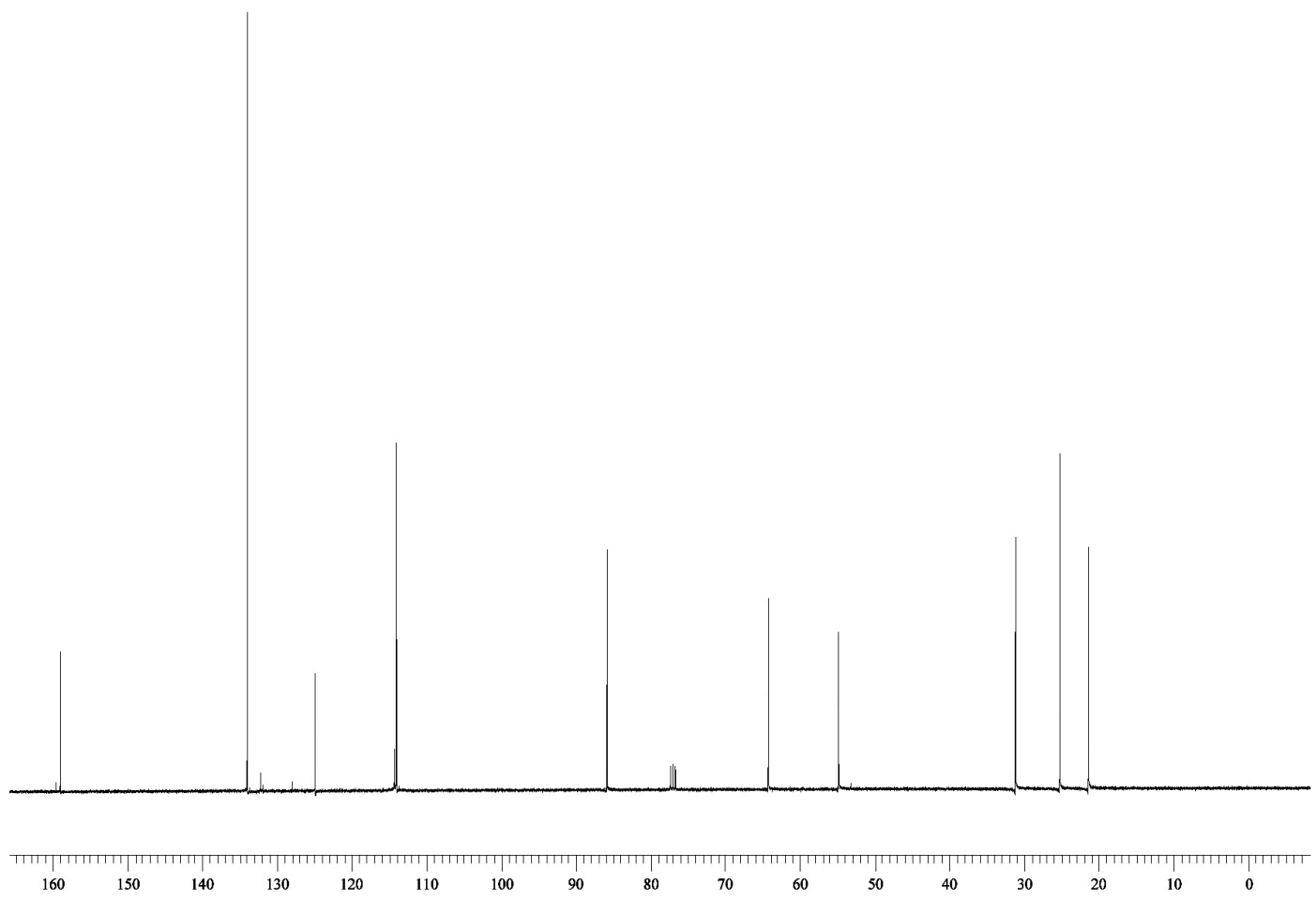

Figure S26. ${ }^{13} \mathrm{C}$ NMR spectra of compound $\mathbf{5 c}$ in $\mathrm{CDCl}_{3}$ at $100 \mathrm{MHz}$. 


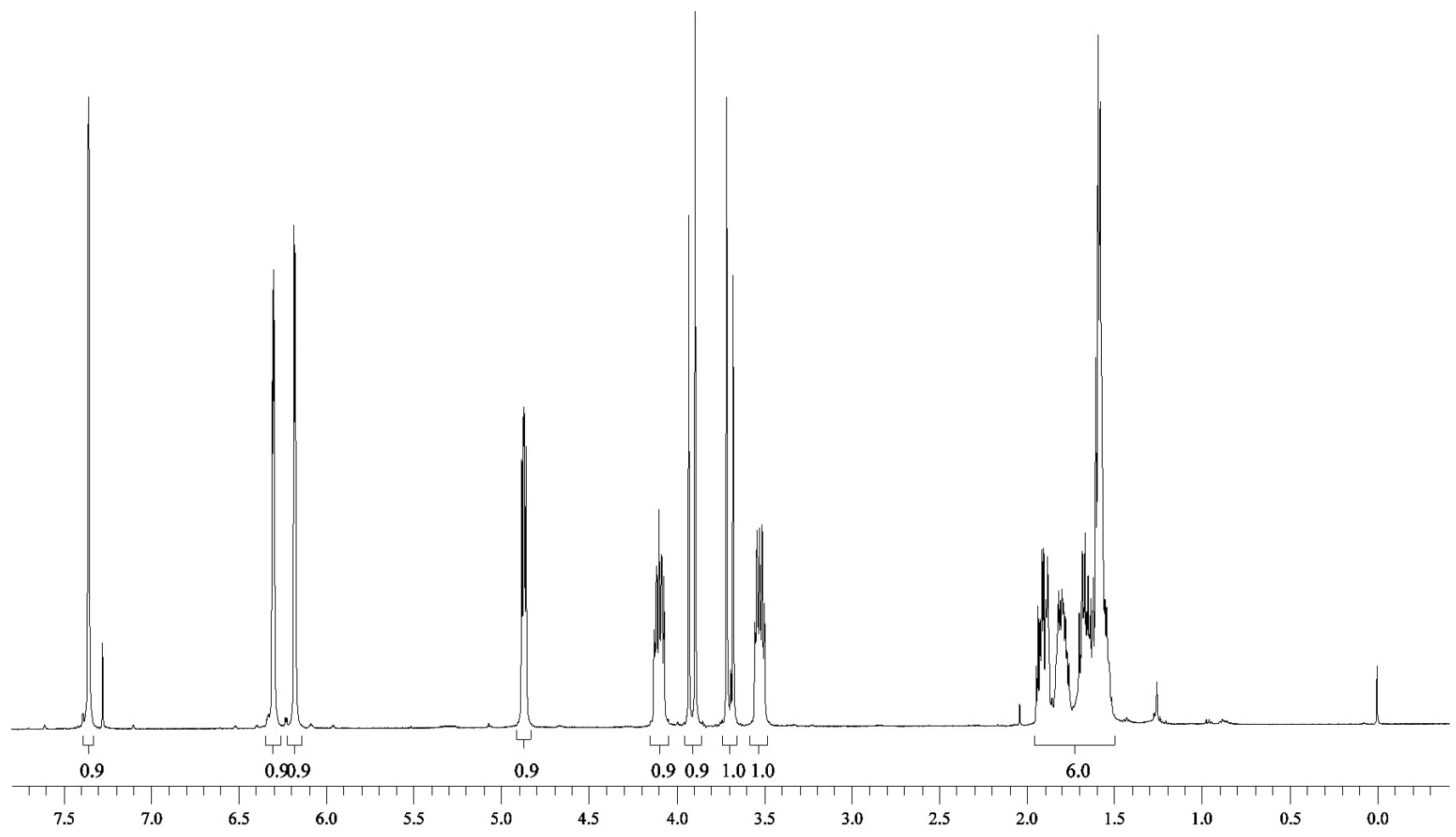

Figure S27. ${ }^{1} \mathrm{H}$ NMR spectra of compound $\mathbf{5 d}$ in $\mathrm{CDCl}_{3}$ at $400 \mathrm{MHz}$.

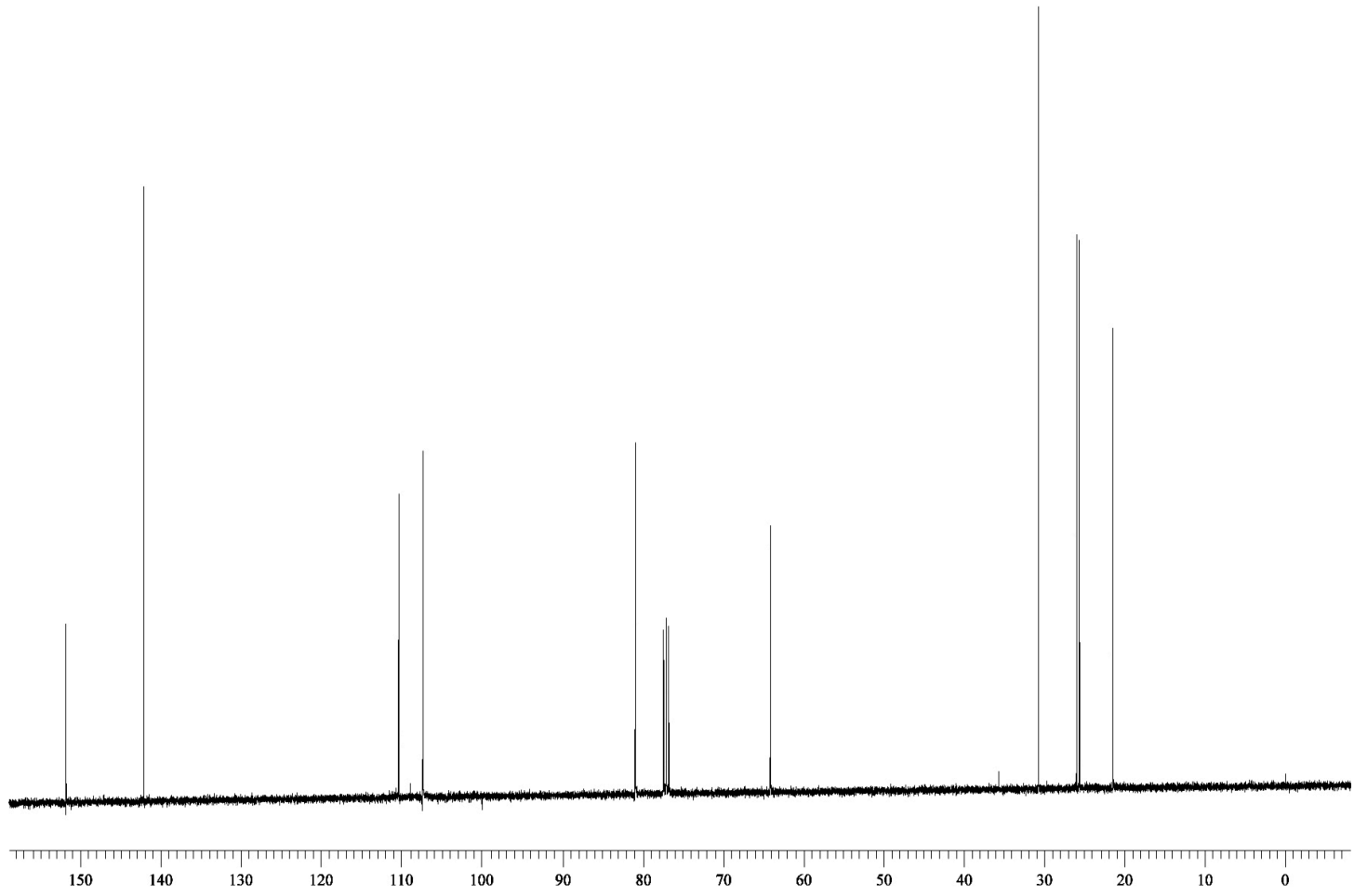

Figure S28. ${ }^{13} \mathrm{C}$ NMR spectra of compound $\mathbf{5 d}$ in $\mathrm{CDCl}_{3}$ at $100 \mathrm{MHz}$. 


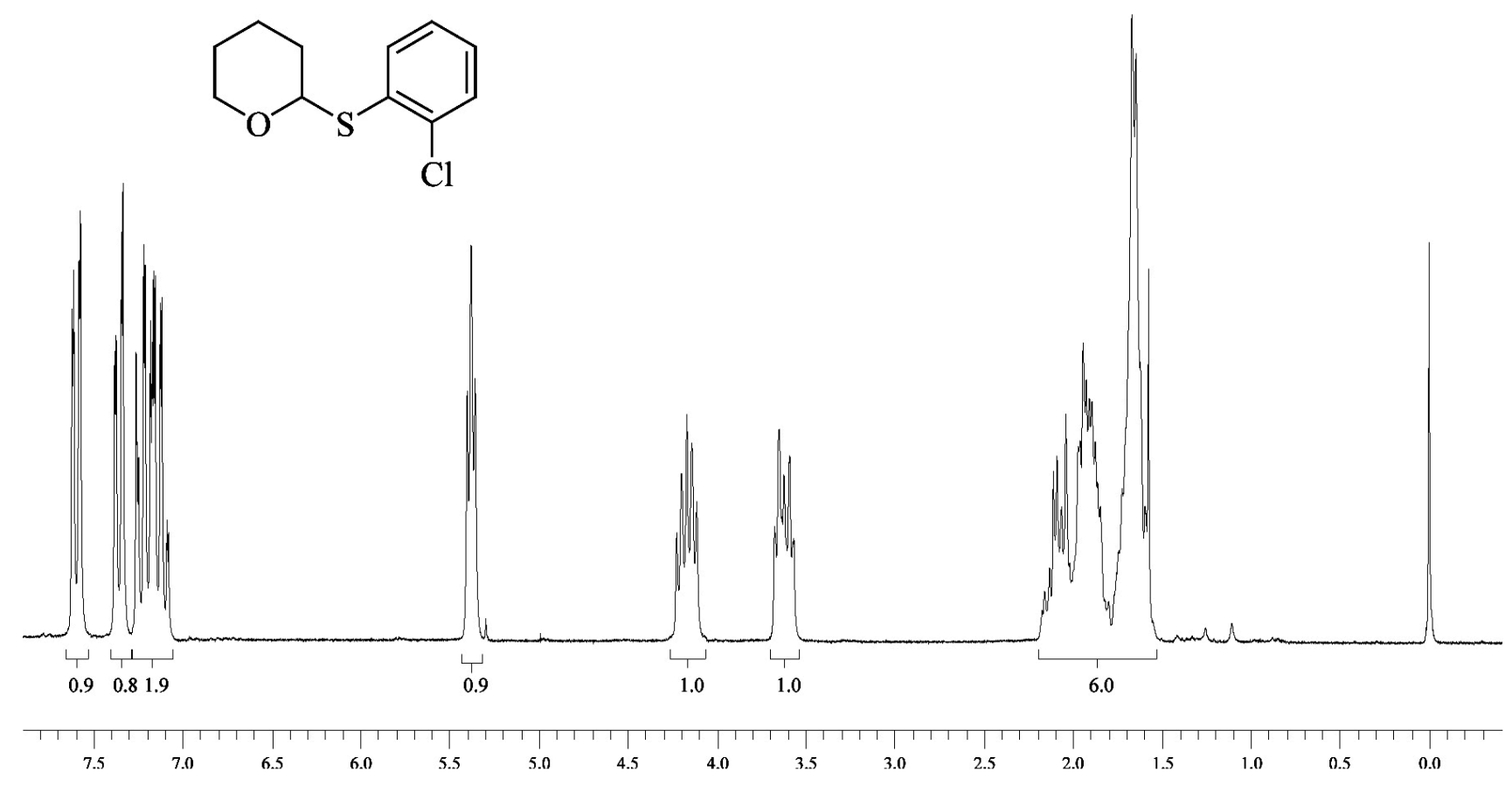

Figure S29. ${ }^{1} \mathrm{H}$ NMR spectra of compound $\mathbf{5 e}$ in $\mathrm{CDCl}_{3}$ at $400 \mathrm{MHz}$.
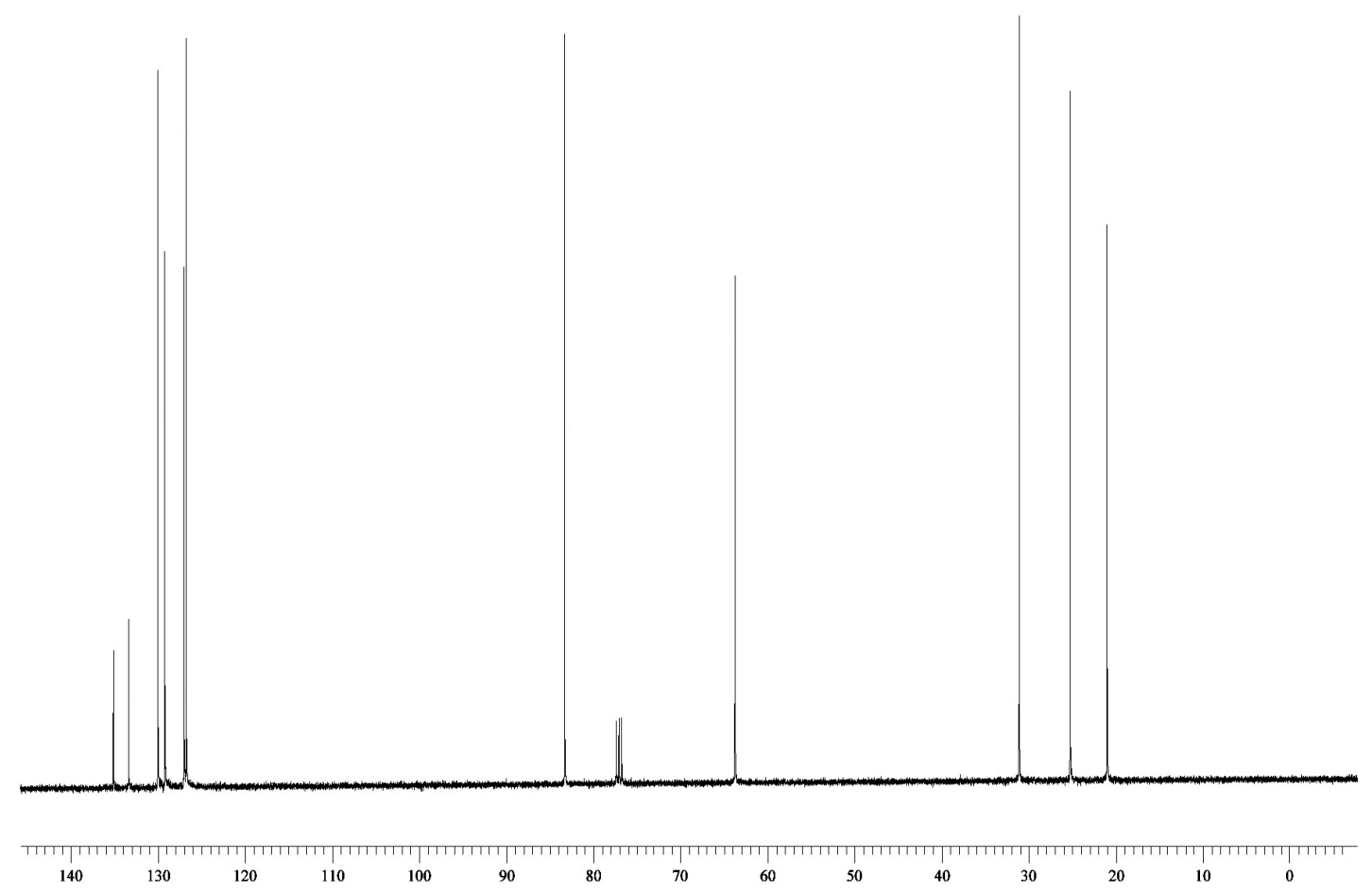

Figure S30. ${ }^{13} \mathrm{C}$ NMR spectra of compound $\mathbf{5 e}$ in $\mathrm{CDCl}_{3}$ at $100 \mathrm{MHz}$. 